\title{
Evolution of the Accretion Disk-Corona during the Bright Hard-to-soft State Transition: A Reflection Spectroscopic Study with GX 339-4
}

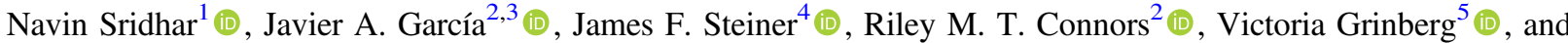 \\ Fiona A. Harrison ${ }^{2}$ \\ ${ }^{1}$ Department of Astronomy, Columbia University, 550 W 120th Street, New York, NY 10027, USA; navin.sridhar@columbia.edu \\ ${ }^{2}$ Cahill Center for Astronomy and Astrophysics, California Institute of Technology, Pasadena, CA 91125, USA \\ ${ }^{3}$ Dr. Karl Remeis-Observatory and Erlangen Centre for Astroparticle Physics, Sternwartstr. 7, D-96049 Bamberg, Germany \\ ${ }_{5}^{4}$ MIT Kavli Institute for Astrophysics and Space Research, MIT, 70 Vassar Street, Cambridge, MA 02139, USA \\ ${ }^{5}$ Institute für Astronomie and Astrophysik (IAAT), Universität Tübingen, Sand 1, D-72076 Tübingen, Germany \\ Received 2019 September 9; revised 2019 December 18; accepted 2019 December 22; published 2020 February 12
}

\begin{abstract}
We present the analysis of several observations of the black hole binary GX 339-4 during its bright intermediate states from two different outbursts (2002 and 2004), as observed by the RXTE/Proportional Counter Array. We perform a consistent study of its reflection spectrum by employing the RELXILL family of relativistic reflection models to probe the evolutionary properties of the accretion disk including the inner disk radius $\left(R_{\text {in }}\right)$, ionization parameter $(\xi)$, temperatures of the inner disk $\left(T_{\text {in }}\right)$, corona $\left(k T_{\mathrm{e}}\right)$, and its optical depth $(\tau)$. Our analysis indicates that the disk inner edge approaches the innermost stable circular orbit during the early onset of the bright hard state, and that the truncation radius of the disk remains low $\left(<9 R_{\mathrm{g}}\right)$ throughout the transition from hard to soft state. This suggests that the changes observed in the accretion disk properties during the state transition are driven by variation in accretion rate, and not necessarily due to changes in the inner disk's radius. We compare the aforementioned disk properties in two different outbursts with state transitions occurring at dissimilar luminosities and find identical evolutionary trends in the disk properties, with differences only seen in the corona's $k T_{\mathrm{e}}$ and $\tau$. We also perform an analysis by employing a self-consistent Comptonized accretion disk model accounting for the scatter of disk photons by the corona, and measure the low inner disk truncation radius across the bright intermediate states, using the temperature-dependent values of the spectral hardening factor, thereby independently confirming our results from the reflection analysis.
\end{abstract}

Unified Astronomy Thesaurus concepts: High energy astrophysics (739); Compact objects (288); Black hole physics (159); Low-mass x-ray binary stars (939); Accretion (14); X-ray astronomy (1810); Spectroscopy (1558); Astronomy data modeling (1859); X-ray sources (1822); General relativity (641); Gravitation (661); Astrophysical black holes (98)

\section{Introduction}

One of the unsettled issues pertaining to black hole X-ray binaries (BHXBs) is the origin of state transitions (Tananbaum et al. 1972) seen during outbursts and the associated evolution of the various intrinsic physical parameters of the accretion disk (Homan \& Belloni 2005; Remillard \& McClintock 2006; Fender \& Belloni 2012). These state transitions are accompanied by changes in the overall luminosity of the source, relative flux of thermal and nonthermal emission, shape of the photon energy spectrum, onset of timing variability features in the light curves (quasi-periodic oscillations; van der Klis 1989), and possible launching of ballistic radio jets and collimated outflows (Mirabel \& Rodríguez 1999; Fender et al. 2004, 2009). Typically, a given source will spend longer periods between outbursts in quiescence, i.e., in a state where little activity is seen across the electromagnetic spectrum. The flux of the source is dominated by hard, nonthermal X-ray photons during the early onset as well as the late-time fading of an outburst and constitute what is called the hard state (with photon index $\Gamma \lesssim 1.8$ ). The nonthermal $\mathrm{X}$-ray emission is presumed to be arising from unsaturated Comptonization of disk photons by a cloud of Maxwellian electrons (called the "corona"; Bisnovatyi-Kogan \& Blinnikov 1976; Sunyaev \& Truemper 1979). In between these two hard states lie the soft and the intermediate states. The soft state, usually observed during the midoutburst period, has its spectra well described by thermal emission from the disk $(\sim 1 \mathrm{keV})$ and occasionally by an additional power-law component with $\Gamma \gtrsim 2.5$. The more complicated intermediate states are characterized by a relative steepening of the nonthermal power-law component compared to the hard state, the appearance of a thermal disk emission component, marked changes to the characteristic frequencies and fractional root mean square (rms) of the power density spectrum, and the launching of relativistic jets (refer to Homan \& Belloni 2005 for more details on intermediate states and its subclassifications into hard and soft intermediate states). Comprehensive studies of stellar BHXBs are therefore critical to deepen our understanding of black hole disks, coronae, jets, their relationships, and their evolution during the state transitions. From the HEASARC RXTE archive, we have chosen GX 339-4 as our source for probing the disk and coronal evolutionary behavior, as this source is highly dynamic and has undergone more than a dozen outbursts since its discovery (Fender et al. 2004).

GX 339-4 is an archetypal BHXB, whose first outburst was reported in 1973, and has been extensively studied since its discovery (e.g., Markert et al. 1973; Makishima et al. 1986; Miyamoto et al. 1991; Méndez \& van der Klis 1997; Corbel et al. 2003; Hynes et al. 2003; Gallo et al. 2004; Zdziarski et al. 2004; Belloni et al. 2005; Plant et al. 2015). It is known to be a part of a binary system located at a distance $(d)$ of $5 \mathrm{kpc}<d<15 \mathrm{kpc}$ (Hynes et al. 2004; Zdziarski et al. 
2004; Parker et al. 2016), with an orbital period of $\sim 1.7$ days (Hynes et al. 2003), and whose inclination is not well known (Cowley et al. 2002; Zdziarski et al. 2019). A relatively recent dynamical study of the binary by Heida et al. (2017) has placed a constraint on the mass of GX 339-4 between $2.3 M_{\odot}$ and $9.5 M_{\odot}$. This uncertainty stems from the lack of precise knowledge of the system's inclination, which need not be the same as the inclination of the accretion disk. The inclination of the accretion disk, on the other hand, has been found to have an intermediate value $\left(30^{\circ}-60^{\circ}\right)$ via different methods, namely X-ray reflection studies (Fürst et al. 2015; García et al. 2015; Basak \& Zdziarski 2016; Parker et al. 2016), and based on the separation of the double-peaked emission lines (Wu et al. 2001). This source has also exhibited ballistic jets observed in the radio band, which are typically associated with the intermediate or steep power-law (SPL) states (Miyamoto \& Kitamoto 1991; Gallo et al. 2004; Steiner et al. 2013).

García et al. (2015) perform the X-ray reflection spectroscopic analysis of the hard-state spectra of GX 339-4 from the $R X T E$ archive, spanning a wide range of luminosities- $1.6 \%$ to $17 \%$ of the Eddington luminosity $\left(L_{\text {Edd }}\right)$. With an assumed black hole spin of $a_{\star}=0.998$, they find the inner edge of the disk reaching $\sim 2 R_{\mathrm{g}}$ (the gravitational radius, $R_{\mathrm{g}}$, is given by $2 G M / c^{2}$, where $M$ is the black hole's mass), as the luminosity increases from $1.6 \% L_{\mathrm{Edd}}$ to $17 \% L_{\mathrm{Edd}}$, and for the assumption of the disk at the innermost stable circular orbit (ISCO), they also constrain the spin of the source at $a_{\star}=0.95_{-0.05}^{+0.03}$. We choose to study the bright intermediate states of this source because it would be a natural segue from the hard-state work of García et al. (2015) into the softer states, and also for the following reasons. Evidence for the intermediate states was reported for the first time by Méndez \& van der Klis (1997) using the EXOSAT observation of the source. During the intermediate states, the spectral hardness has an intermediate value-larger than the soft state's and smaller than the hard state's spectral hardness. What makes it particularly interesting is the possible non-monotonicity in the evolution of parameters governing the bright intermediate state transition, and studying it may provide clues on how sources in general transition between hard and soft states.

Of all the physical parameters of GX 339-4, the one that is highly debated is the physical evolution of the truncated accretion disk's inner radius $\left(R_{\mathrm{in}}\right.$; refer to Appendix A of Basak $\&$ Zdziarski 2016 for a comparison of different $R_{\text {in }}$ estimates), especially during the hard state. Based on Suzaku data of GX 339-4, Tamura et al. (2012) had reported that the optically thick disk is truncated significantly away from the ISCO during the hard-intermediate state. During the hard state, Meyer et al. (2000) had predicted that thermal conduction of heat from the corona will cause the inner disk to evaporate, leading to an optically thick and geometrically thin disk that is truncated at some significant distance away from the black hole. While there is evidence for truncation during the hard state (Mahmoud et al. 2019) and at luminosities below $0.1 \%$ of the Eddington limit $\left(L_{\text {Edd }}\right)$ for GX 339-4 (Tomsick et al. 2009), measurements of the reflection component in the bright hard state $\left(>5 \% L_{\mathrm{Edd}}\right)$ have led to estimates of inner radii very close to the ISCO for several sources, including GX 339-4 (García et al. 2015, 2019; Steiner et al. 2017; Wang-Ji et al. 2018). While there exists abundant evidence for a truncated disk at the low/hard state and the disk extending up to the ISCO by the time the source reaches the bright soft state (Gierliński \& Done 2004; Penna et al. 2010; Steiner et al. 2010; Zhu et al. 2012), the evolution of the disk and the coronal parameters, especially the inner disk's radius during the bright intermediate states, is not well understood.

The reflection component of the X-ray spectrum results from the reprocessing of the Compton upscattered nonthermal X-ray photons in the optically thick accretion disk. This reflected radiation is adorned with several prominent features including the recombination continuum, fluorescent emission lines, and absorption edges. These features provide information on the physical state of the matter and its composition in the strong gravitational regime near the black hole, and serves as a robust means of studying conditions near the event horizon. In particular, modeling the distortion in the prominently seen $\mathrm{Fe}$ K fluorescent line due to Doppler effects, gravitational redshift and light bending can contribute to the understanding of the spin of the black hole, the accretion disk's inner edge radius, and its inclination. In particular, having the spin fixed to a constant value of the Thorne maximum ( $a_{\star}=0.998$; Thorne 1974), one can estimate the minimum truncation of the inner radius of the disk with much better sensitivity (refer to Bardeen et al. 1972 for a description of particle orbits in Kerr metric and the relation between the black hole's spin and the ISCO).

Although a study of GX 339-4 during the bright hard-to-soft state transition has been performed by Del Santo et al. (2008), a probe of the reflection spectrum during this state transition remains relatively underexplored. Using a version of the relativistic reflection model (relcillcp; Dauser et al. 2014; García et al. 2014a) that includes a physical Comptonization kernel (nthComp; Zdziarski et al. 1996), we derive constraints on the evolution of the system's parameters like the inner radius of the disk, its temperature, the disk ionization state, and the temperature and optical depth of the corona during the transit of the source from the bright hard to soft states of two outbursts with different transition luminosities (the 2002-2003 outburst with higher flux and the 2004-2005 outburst with lower flux), as observed by RXTE (refer to Plant et al. 2014 for a reflection study of the source across various state transitions using a different model and assumptions from us). We also perform an analysis of the continuum spectra from the bright intermediate state transition by employing a selfconsistent Comptonized accretion disk model. This model accounts for the scattering of the disk photons by the corona to arrive at the disk and coronal properties independent of the reflection modeling and to compare the results from one method against the other.

This paper is organized as follows: in Section 2, we describe the observation, data selection, and reduction procedure; in Section 3, we provide the detailed analysis procedure, results from our analysis of reflection and continuum spectra, and the corresponding statistical (Markov Chain Monte Carlo; MCMC) analysis. In Section 4, we discuss the broad implications of our results, and summarize the same in Section 5. A test of the potential of RXTE/Proportional Counting Array (PCA) and the RELXILL model to detect large inner disk truncation values is provided in Appendix A.

\section{Observations and Data Reduction}

We select our sample of the bright intermediate state observations of the Galactic X-ray binary GX 339-4 from the huge inventory of data that RXTE (Swank 1999) had amassed during its lifetime. Figure 1 shows four distinct 


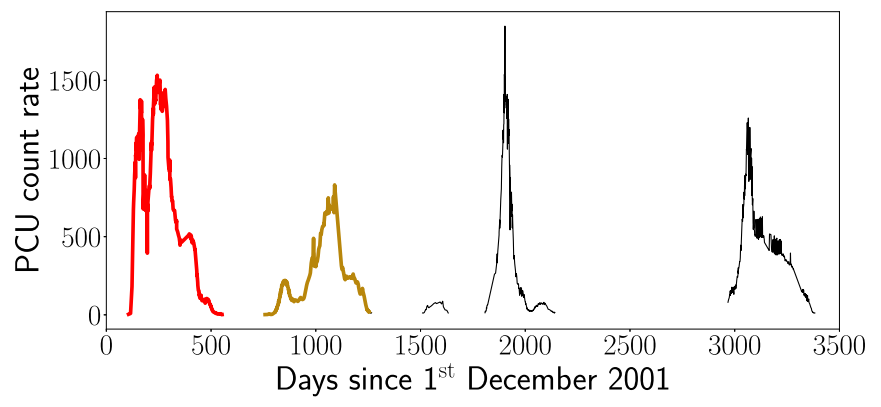

Figure 1. $R X T E / P C A$ light curve of GX 339-4 showing its four prominent outbursts over a period of $\sim 10 \mathrm{yr}$. The vertical axis shows the count rate (intensity) and plotted on the horizontal axis is the days that have passed since 1 December 2001 (MJD 52244). The outbursts highlighted in red and golden-brown colors are the ones considered for the analysis presented in this paper. We call the red one the "2002-2003 outburst" and the golden-brown one as the "2004-2005 outburst."

outbursts of GX 339-4 spanning $\sim 10 \mathrm{yr}$, as observed by $R X T E / P C A$. We define here the bright intermediate state to be the one bridging the brightest hard and soft states, with hardness ratio (HR) values in the range $0.05<\mathrm{HR}<0.8$, where HR is the ratio of the source counts at the $8.6-18.0 \mathrm{keV}$ to $5.0-8.6 \mathrm{keV}$ energy band. This region is seen in Figure 2 in the top horizontal row of each outburst. Figure 2 is called the hardness-intensity diagram (HID), where the X-ray hardness (denoted above as HR) is plotted against the observed count rate (intensity). In the HID, outbursts in 2002-2003, 2006-07, and 2010-11 can be seen to trace overlapping tracks across the bright intermediate states. We choose the earlier outburst as representative of the three of them. The 2004-2005 outburst clearly traces a different path across the bright intermediate states with a much smaller luminosity compared to the other three outbursts. So, we also choose observations from the 2004-2005 outburst for our analysis to test whether there are any significant differences seen in the disk parameters of this outburst from the rest. The 2002-2003 and the 2004-2005 outbursts are highlighted in the $\sim 10 \mathrm{yr}$ light curve of the source (Figure 1) with red and golden-brown colors respectively. The individual pointings from the bright intermediate states of the 2002-2003 and 2004-2005 outbursts that are considered for the reflection-fitting procedure are listed in Table 1.

The PCA (Jahoda et al. 2006) on board the RXTE mission comprises five nearly identical proportional counting units (PCUs). The data from all five PCUs are reduced and background subtracted following the procedures described in McClintock et al. (2006). Data are taken in standard-2 mode, which provides coverage of the PCA bandpass every $16 \mathrm{~s}$ with exposure times ranging from $300 \mathrm{~s}$ to $5000 \mathrm{~s}$. Background spectra are derived using the PCABACKEST tool with pca_bkgd_cmvle_eMv20111129.mdl as the background model. Response files are generated using the tool PCARMF (version 11.7) considering the energy-to-channel conversion table (version e05v04) as described in Shaposhnikov et al. (2012). Following García et al. (2014b), we apply the tool PCACORR to all the data. This tool corrects for small imperfections in the spectrum, resulting in an increased sensitivity of PCA to faint spectral features. It therefore allows us to consider a much lower systematic uncertainty of $0.1 \%$. Calibration of the PCUs have been found to be uncertain above $45 \mathrm{keV}$, and the residuals seen below $3 \mathrm{keV}$ cannot be

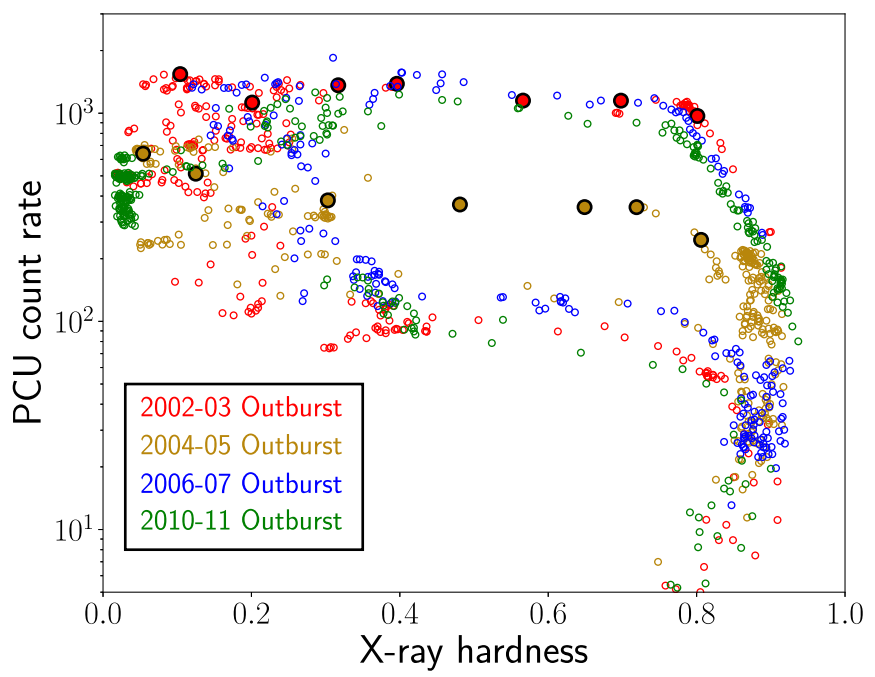

Figure 2. Hardness-intensity diagram (HID) from all RXTE/PCA observations of GX 339-4 spanning $\sim 10 \mathrm{yr}$. Plotted on the horizontal axis is the X-ray hardness, defined as the ratio of the source counts in $8.6-18 \mathrm{keV}$ to the counts in $5.0-8.6 \mathrm{keV}$ energy band, and the vertical axis represents the PCU count rate (intensity). In this paper, we consider the 2002-2003 outburst (red) and the 2004-2005 outburst (golden-brown) for our analysis. Specifically, the interspaced highlighted red and golden-brown circles represent the positions of the observations on the HID that are considered for the reflection analysis.

explained by any plausible spectral features. So, we ignore the spectrum belonging to channels $1-4$ and above $45 \mathrm{keV}$, and include only the rest, for our analysis.

\section{Results}

All of the spectral data analysis and model fitting are performed using heasoft-6.23 and XSPEC version 12.9.1q (Arnaud 1996). All models considered for the analysis described herein include the Galactic absorption effect by implementing the TBabs model (Wilms et al. 2000) with the corresponding abundances and cross sections set according to the Anders \& Grevesse (1989) and Verner et al. (1996) photoelectric cross sections. This model is parameterized by the hydrogen column density, which, for this source, we fix at a value of $5.9 \times 10^{21} \mathrm{~cm}^{-2}$. This value is consistent with the value determined for this source from an analysis of RXTE/ PCA data by García et al. (2015). We start our analysis by modeling the spectra with simple continuum models aimed at visualizing the $\mathrm{Fe} \mathrm{K}$ reflection feature. We then extend our analysis by modeling all the key reflection signatures in the spectrum (i.e., Fe K emission, Fe K edge, and Compton hump) using full-fledged relativistic reflection models. In the next few sections, we describe the various exercises performed with the bright intermediate state data of the source and the corresponding results.

\subsection{Ratio Plots: Model Dependency and Search for Line Broadening}

The aim of this piece of work is to check if the Fe $\mathrm{K}$ reflection feature from the disk at different HR values has any dependence on the chosen model of the Comptonization continuum. To start with, we consider the selected observations from the bright intermediate states of the 2002-2003 outburst (see Section 2 and Table 1) and model them with three simple semiphysical models: 
Table 1

The RXTE/PCA Observation Log of GX 339-4 across the Bright Intermediate States of the 2002-2003 and 2004-2005 Outbursts (Highlighted by the Red and Golden-brown Circles in Figure 2) Considered for the Reflection Analysis

\begin{tabular}{|c|c|c|c|c|c|}
\hline Outburst & ObsId & Start Time & Exposure (s) & Counts $\left(\times 10^{5}\right)$ & HR \\
\hline \multirow{6}{*}{ 2002-2003 } & 40031-03-01-00 & 2002 Apr 18 02:42:59 & 1856 & 17.72 & 0.80 \\
\hline & 70109-01-06-00 & 2002 May 6 20:12:04 & 1920 & 21.38 & 0.70 \\
\hline & 70110-01-10-00 & 2002 May 8 11:58:05 & 976 & 10.53 & 0.56 \\
\hline & 70110-01-12-00 & 2002 May 16 12:50:26 & 1248 & 14.94 & 0.30 \\
\hline & 40031-03-03-04 & 2002 May 20 08:42:45 & 1632 & 15.69 & 0.20 \\
\hline & 70110-01-33-00 & 2002 Jul 28 19:54:20 & 880 & 11.54 & 0.10 \\
\hline & 90704-01-01-00 & 2004 Aug 9 10:50:28 & 3168 & 10.88 & 0.72 \\
\hline & 60705-01-69-01 & 2004 Aug 11 23:55:57 & 848 & 2.88 & 0.65 \\
\hline 2004-2005 & 60705-01-70-00 & 2004 Aug 13 23:07:57 & 720 & 2.44 & 0.48 \\
\hline & 90704-01-03-00 & 2004 Aug 18 10:39:48 & 2736 & 9.45 & 0.30 \\
\hline & $60705-01-76-00$ & 2004 Sep 24 15:58:17 & 656 & 3.02 & 0.13 \\
\hline & 90118-01-10-01 & 2004 Oct 28 12:08:33 & 2752 & 15.81 & 0.05 \\
\hline
\end{tabular}

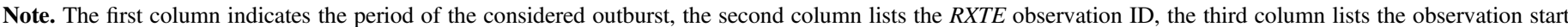

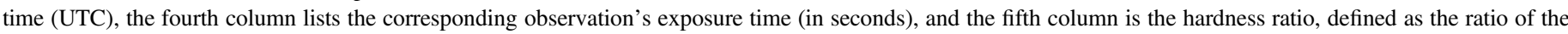
source count rate in the $8.6-18.0 \mathrm{keV}$ energy band to the count rate in the $5.0-8.6 \mathrm{keV}$ energy band.

1. $\mathrm{TBabs} *$ smedge (diskbb+cutoffpl+Gaussian)

2. $\mathrm{TBabs} *$ smedge (diskbb+powerlaw+Gaussian)

3. TBabs*smedge (diskbb+nthComp+Gaussian)

where the galactic absorption is modeled with TBabs, the multicolored disk blackbody emission is modeled with diskbb (Mitsuda et al. 1984; Makishima et al. 1986), the Fe $\mathrm{K}$ reflection feature is modeled with a Gaussian, and the smeared absorption edge at $\sim 7.1 \mathrm{keV}$ is modeled with smedge (Ebisawa 1991, implemented by Frank Marshall). Each model is different from the others in the choice of the implementation for modeling the nonthermal emission component. Models 1 and 2 use the phenomenological cutoff powerlaw (cutoffpl) and simple powerlaw models, respectively, whereas model 3 employs the physically motivated nthComp (developed by Zdziarski et al. 1996 and extended by Życki et al. 1999) model to describe the Comptonized component.

In all three models, the smedge and Gaussian widths are frozen at $7 \mathrm{keV}$ and $0.01 \mathrm{keV}$, respectively, and the index for photoelectric cross section in the smedge component is set to its standard value of -2.67 . After fitting the spectra with these models, we retain the best-fit parameters and remove the respective Gaussian components. These Gaussian-less models are then used to visualize the $\mathrm{Fe} \mathrm{K}$ reflection feature from the data/model ratio plots. This approach validates that the profile of the $\mathrm{Fe} \mathrm{K}$ reflection feature is insensitive to the model used to describe the Comptonized component of the spectrum. Reflection features during the intermediate states have also been observed by Plant et al. (2014).

After establishing the insensitivity of the profile of the $\mathrm{Fe} \mathrm{K}$ reflection feature to different Comptonization models, we choose to model the spectrum with TBabs*smedge (diskbb $+n$ thComp+Gaussian), and look out for any broadening in the reflection features, as we progress across a wide range of HR values. We choose this model because nthComp, unlike the powerlaw components, is a physically motivated model in describing the continuum shape from thermal Comptonization. Using this model, data/model ratio plots are recreated for observations in the bright hard state of the 2002-2003 outburst, following the procedures mentioned above. Figure 3 shows the

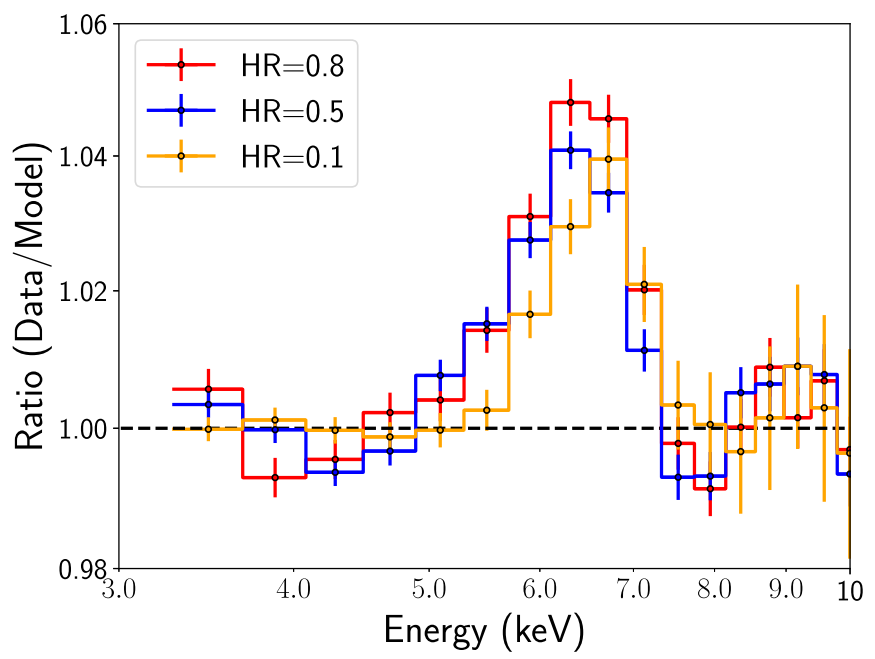

Figure 3. Data/model ratio plots produced at different hardness values across the bright intermediate states of the 2002-2003 outburst of GX 339-4 using the model TBabs*smedge (diskbb+nthComp). No significant change in the width of the $\mathrm{Fe} \mathrm{K}$ line is observed with decreasing spectral hardness.

ratio plot depicting the $\mathrm{Fe} \mathrm{K}$ emission feature, plotted for three different representative values of $\operatorname{HR}(\simeq 0.8,0.5,0.1)$ across the bright intermediate states. Should the inner disk radius be significantly different between the soft and hard states, then the width of the iron line should also be different (see Appendix B). However, no such significant change in the broadening of the emission line can be seen.

\subsection{Fitting with the Reflection Model}

The spectrum of a typical BHXB can be described well by the combination of a quasi-thermal blackbody emission due to the radiatively efficient accretion disk (Shimura \& Takahara 1995), a nonthermal power-law component due to Compton upscattering of the thermal disk photons by a hot cloud of electron-positron pairs (Zdziarski \& Gierliński 2004, called Corona), and the reflection component of the nonthermal emission by the optically thick accretion disk (Ross \& 
Fabian 1993), if seen. In our analysis, we model the thermal blackbody component of the spectrum using the diskbb model and the nonthermal emission using the nthComp model. Following García et al. (2015), we model the reflection features with a combination of relativistic disk reflection and a distant reflector for the narrow emission-line component.

The spectra of GX 339-4 have been shown to exhibit reflection features in their hard (García et al. 2015) and soft states (Miller et al. 2004; Reis et al. 2008). In the previous section (Section 3.1), we showed the presence of the Fe K reflection feature across the bright intermediate states in the spectra. We account for that feature and other possible reflection components (namely, the $\mathrm{Fe} \mathrm{K}$ absorption edge, Compton hump) by modeling them with the physically rigorous RELXILL family of relativistic reflection models. The relativistically blurred reflection component is described using relxillCp (Dauser et al. 2014; García et al. 2014a) and the narrow distant reflection from the disk is described using the xillverCp (García \& Kallman 2010; García et al. 2013) model. The default shape of the illuminating continuum of the RELXILL and XILLVER models is a simple power law with an exponential cutoff at higher energies. However, in our model, we describe the nonthermal emission by the physically motivated nthComp component, and hence, the relxillCp and xillverCp flavors of the reflection models are used, which calculate the reflection spectrum by self-consistently using the nthComp continuum. The total model including Galactic absorption is given by TBabs (diskbb+nthComp +relxillcp+xillvercp).

In order to obtain better constraints of the key unknown parameters we are interested in (e.g., $R_{\text {in }}, T_{\text {in }}$, electron temperature $k T_{\mathrm{e}}$ ), we freeze the other parameters to their previously measured values. The hydrogen absorption column density is fixed at $N_{\mathrm{H}}=5.9 \times 10^{21} \mathrm{~cm}^{-2}$, the disk inclination is frozen at an intermediate value of $i=45^{\circ}$, and the iron abundance $A_{\mathrm{Fe}}$ in the accretion disk at $A_{\mathrm{Fe}}=5$ (in solar units). These numbers are consistent with the values of the corresponding parameters determined from the reflection analysis of $R X T E / P C A$ and NUSTAR data of this source by García et al. (2015), Fürst et al. (2015), and Parker et al. (2016). These are the intrinsic parameters of the system and are not expected to change over the course of outbursts. The spin value measurements of GX 339-4 by Miller et al. (2004), Reis et al. (2008), Miller et al. (2008), García et al. (2015), and Parker et al. (2016) have indicated that GX 339-4 is a black hole with a very high spin, close to the Kerr value (Kerr 1963). Therefore, we fix the spin of the black hole at the limiting value of $a_{*}=0.998$, so that a wide range of $R_{\text {in }}$ can be fully explored. One of the available parameters of the reflection models is the outer radius of the accretion disk $\left(R_{\text {out }}\right)$. We freeze it to a value large enough $\left(R_{\text {out }}=10^{3} R_{\mathrm{g}}\right)$ such that our measurements are not sensitive to its setting. This frozen value of $R_{\text {out }}$ is much smaller than the actual radius of the outer disk and only corresponds to that region of the disk that is relevant for X-ray reflection. The blurred and the unblurred reflection components (relxillcp and xillverCp) each accept a parameter for the reflection fraction (allowing for a thermal Comptonization continuum), which is defined as the ratio of the intensity emitted toward the disk to that of the intensity escaping to infinity, in the frame of the primary source (see Dauser et al. 2016 for more details). As we include an $n$th Comp component explicitly accounting for the thermal Comptonization continuum, we freeze the reflection fraction parameter $\left(R_{\mathrm{f}}\right)$ to -1 , so that the relxillCp component calculates only the reflected emission and the overall model does not have two identical continuum components.

Some of the underlying assumptions of our analysis are as follows. Nearly neutral narrow $\mathrm{Fe} \mathrm{K}$ lines have earlier been reported in bright Galactic binaries (Parker et al. 2015). We therefore assume that the unblurred, distant reprocessing material is relatively cold and nearly neutral, and hence the log of the ionization parameter $(\log \xi)$ corresponding to the xillverCp component is frozen at zero (where the ionization parameter is given by $\xi=L / n R^{2}$, with $L$ being the ionizing luminosity, $n$ being the gas density, and $R$ being the distance to the ionizing source). This assumption is also adopted by García et al. (2015) for this source. For these fits, we also assume that the disk obeys the canonical emissivity profile, $\epsilon \propto r^{-q}$, where $q=3$ is the assumed value of emissivity index (Fabian et al. 1989).

In addition to freezing some of the parameters to previously measured values, we also tie relevant parameters between different components of the model. For instance, the nthComp component accounts for the low-energy rollover due to the seed photons that originate from the accretion disk. Hence, we tie the $n$ thComp input seed photon temperature $\left(k T_{\mathrm{bb}}\right)$ to the inner disk temperature ( $T_{\text {in }}$ of the diskbb component). The previously mentioned assumption of a thermally Comptonized illuminating spectrum allows us to tie relevant parameters from the nthComp component, like spectral index and electron temperature $\left(k T_{\mathrm{e}}\right)$, with the ones in the relxillcp component. Additionally, the values for the $A_{\mathrm{Fe}}$, asymptotic powerlaw index $\Gamma$, disk inclination, and the input continuum in $\mathrm{xillverCp}$ are also linked with those of the broad reflection component (relxillcp), as we find no empirical need to decouple these components' parameters.

With this setup, we simultaneously fit for the inner disk temperature $\left(T_{\mathrm{in}}\right)$, photon index $(\Gamma)$, electron temperature in the corona $\left(k T_{\mathrm{e}}\right.$, with a hard upper limit at $\left.400 \mathrm{keV}\right)$, inner disk radius $\left(R_{\mathrm{in}}\right), \log$ of the disk ionization parameter $(\log \xi)$, and the normalization values of the respective model components. The total model flux at different energy ranges is calculated using the flux command in XSPEC, and the flux of the individual model components is evaluated by convolving the component, cflux (in XSPEC notation), with the required model components (e.g., with relxillCp and nthComp, used to calculate the reflection strength $R_{\mathrm{s}}$, as mentioned in the upcoming sections). The spectral data, best-fit model, underlying model components, and the corresponding residuals $(\Delta \chi)$ from the best fit can be seen in Figure 4. In the next couple of sections, we describe in detail the resultant best-fit parameters attained for the considered observations by fitting with the model described above. The best-fit parameters and the $90 \%$ confidence intervals are obtained from the distributions of parameters, derived from performing the MCMC analysis (see Section 3.2.3 for more details).

\subsubsection{The 2002-2003 Outburst (Higher Flux)}

The HID of GX 339-4 during 2002-2011 (see Figure 2) shows three outbursts (2002-2003, 2006-07 and 2010-11) tracing similar paths in the spectral hardness-luminosity space. Out of them, we choose the 2002-2003 outburst as a representative outburst of that group and perform reflection analysis. We begin by modeling the spectra from the bright intermediate states of 2002-2003 outburst in a temporal 


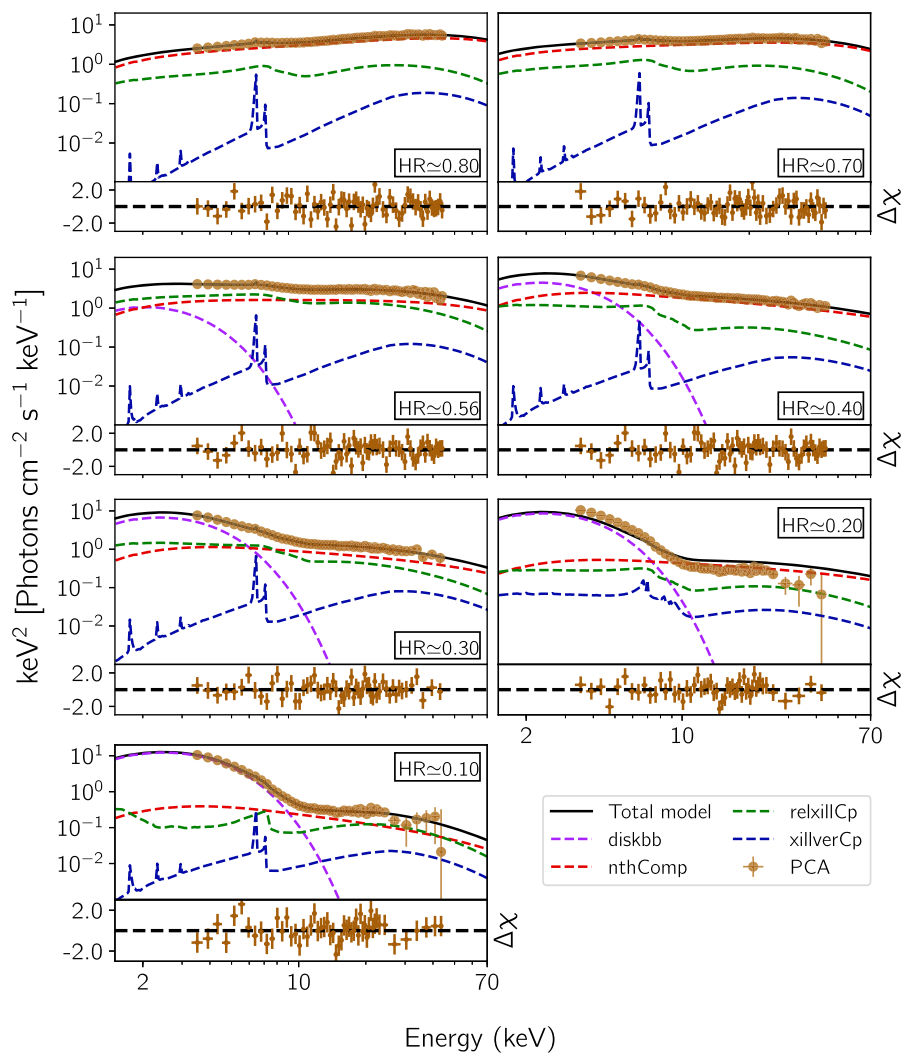

(a) 2002-2003 Outburst
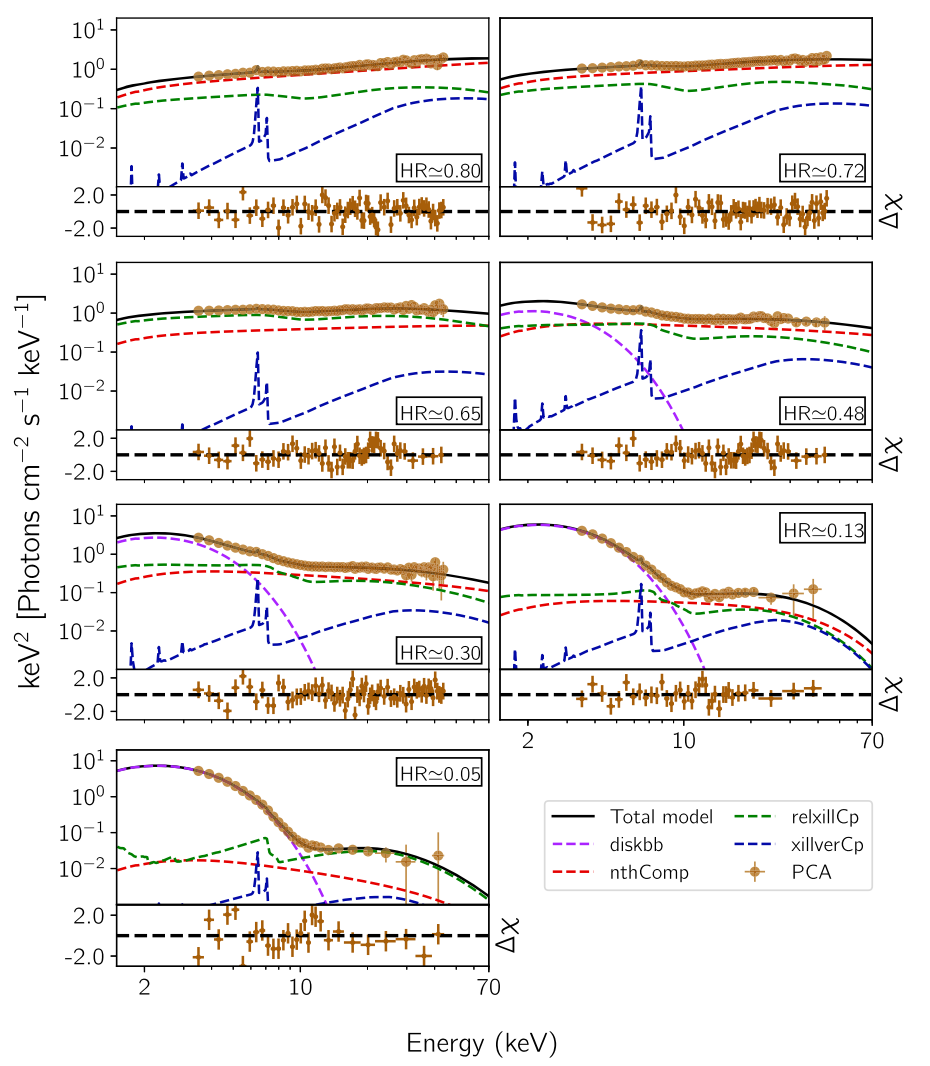

(b) 2004-2005 Outburst

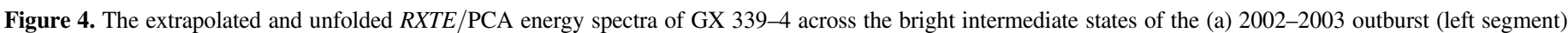

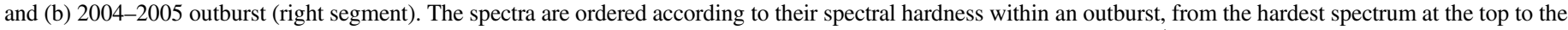

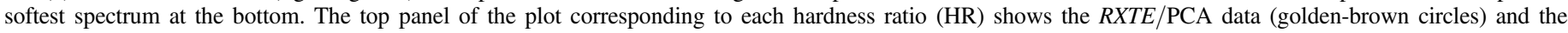

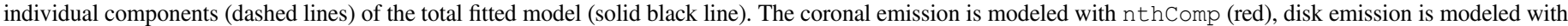

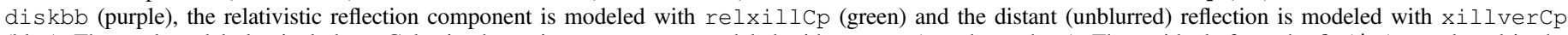

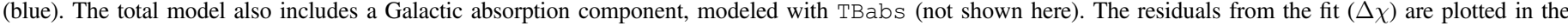
bottom panel of each spectral plot.

sequence, starting with the hard-state $(\mathrm{HR} \simeq 0.8)$ spectrum and gradually proceeding toward the soft state $(\mathrm{HR} \simeq 0.1$; see Table 1 and red markers in Figure 2). The best-fit parameters and the $90 \%$ confidence intervals obtained by fitting the 2002-2003 observations with our reflection model are listed in Table 2.

During the analysis, we notice that the hardest two spectra considered here $(\mathrm{HR} \simeq 0.8$ and 0.7$)$ do not exhibit a significant thermal emission component and including a diskbb component with free parameters is not required. The absence of a distinct blackbody emission component in the spectra can be seen in the top two panels of Figure 4(a). Therefore, we freeze the normalization parameter of the diskbb component to 0 , and as a result, we are unable to arrive at a good constraint on the temperature of the inner disk. As the outburst progresses toward the intermediate states $(\mathrm{HR} \gtrsim 0.56$ in our selection of observations), we see a need for the thermal blackbody component in order to describe the spectra well, and therefore include the diskbb component, and fit for its normalization as a free parameter. In all the forthcoming fits with softer spectra, we observe the $T_{\text {in }}$ and diskbb norm parameters to gradually increase all the way across the bright intermediate states, reaching a value of $T_{\text {in }}=0.87_{-0.01}^{+0.01} \mathrm{keV}$ and norm $=3.81_{-0.11}^{+0.09} \times 10^{3}$, respectively, by the time the spectral hardness reaches a value of $\mathrm{HR} \simeq 0.1$ (see Equation (4) in Section 4.4.2 for a discussion on diskbb norm).
For the cases of $\mathrm{HR} \simeq 0.8$ and 0.7 , the asymptotic spectral indices are found to be $\Gamma=1.68_{-0.01}^{+0.01}$ and $1.83_{-0.01}^{+0.01}$, respectively, which are typical of the hard state (Homan \& Belloni 2005; Remillard \& McClintock 2006). As the spectrum gets softer, the nonthermal tail is seen to get steeper as expected (see the last two panels of Figure 4(a)), thus resulting in an increasing trend in $\Gamma$, as HR decreases. This increase in $\Gamma$ culminates at a value of $2.80_{-0.17}^{+0.07}$ for the spectrum with $\mathrm{HR} \simeq 0.1$. From the fits, we constrain the coronal electron temperatures $\left(k T_{\mathrm{e}}\right)$ to values between $18.2 \mathrm{keV}$ and $25.5 \mathrm{keV}$ during the bright hard to intermediate state transition $(0.8 \geqslant \mathrm{HR} \geqslant 0.56)$. However, the values of the electron temperature cannot be constrained well during the intermediate to soft state transition $(0.56 \geqslant \mathrm{HR} \geqslant 0.1)$.

One of the key physical parameters of the accretion disk that we are interested in to understand its evolution is its inner radius, as the outburst evolves from the bright hard to the bright soft state. In Section 3.1, we qualitatively demonstrated from the unchanging nature of the width of the Fe $\mathrm{K}$ reflection profile that the disk truncation is relatively stable across the bright intermediate state transition. This result is verified by fitting the spectrum with the state-of-the-art reflection model that we employ. Fitting for the $R_{\text {in }}$ parameter of the relxillcp component yields a value of $R_{\text {in }}=6.85_{-2.47}^{+4.15} R_{\mathrm{ISCO}}\left(3.5 R_{\mathrm{g}}-8.9 R_{\mathrm{g}}\right)$ for the hardest spectrum in our sample $(\mathrm{HR} \simeq 0.8) . R_{\text {in }}$ then converges to 
Table 2

Best-fit Parameters and the Corresponding 90\% Confidence Intervals Obtained from Fitting the RXTE/PCA Spectra of GX 339-4 from Its 2002-2003 Outburst Using the Model TBabs (diskbb+nthComp+relxillCp+xillverCp) for Different Hardness Ratio Values across the Bright Intermediate State (see Section 3.2.1)

\begin{tabular}{|c|c|c|c|c|c|c|c|c|}
\hline $\begin{array}{l}\text { Spectral } \\
\text { components }\end{array}$ & Parameters & $\mathrm{HR} \simeq 0.8$ & $\mathrm{HR} \simeq 0.7$ & $\mathrm{HR} \simeq 0.56$ & $\mathrm{HR} \simeq 0.4$ & $\mathrm{HR} \simeq 0.3$ & $\mathrm{HR} \simeq 0.2$ & $\mathrm{HR} \simeq 0.1$ \\
\hline $\begin{array}{l}\text { TBabs } \\
\text { relxillcp } \\
\text { relxillcp } \\
\text { relxillcp } \\
\text { relxillcp } \\
\text { xillvercp }\end{array}$ & $\begin{array}{c}N_{\mathrm{H}}{ }^{\mathrm{a}}\left(\mathrm{cm}^{-2}\right) \\
a_{*}^{\mathrm{b}} \\
i(\mathrm{deg})^{\mathrm{c}} \\
A_{\mathrm{Fe}}{ }^{\mathrm{d}}(\text { Solar }) \\
R_{\mathrm{f}}^{\mathrm{e}} \\
\log (\xi)^{\mathrm{f}}\left(\mathrm{erg} \mathrm{cm} \mathrm{s}^{-1}\right)\end{array}$ & & & 5.9 & $\begin{array}{l}10^{21} \\
98 \\
1\end{array}$ & & & \\
\hline & $\chi_{\nu}^{2 \mathrm{q}}$ & $\frac{72.49}{62}=1.17$ & $\frac{64}{63}=1.02$ & $\frac{57.43}{62}=0.93$ & $\frac{69.48}{62}=1.12$ & $\frac{51.18}{62}=0.83$ & $\frac{52.23}{62}=0.84$ & $\frac{53.24}{62}=0.86$ \\
\hline
\end{tabular}

Notes.

${ }^{\mathrm{a}}$ Hydrogen column density.

${ }^{\mathrm{b}}$ Dimensionless spin parameter of the black hole $\left(a_{\star}=c J / G M^{2}\right.$, where $J$ is the angular momentum of the black hole).

${ }^{\mathrm{c}}$ Disk inclination.

${ }^{\mathrm{d}}$ Iron abundance of the material in the accretion disk.

${ }^{\mathrm{e}}$ Reflection fraction, defined as the ratio of the reflected flux to the flux in the power-law component in the $20-40 \mathrm{keV}$ band.

${ }^{\mathrm{f}} \log$ of the ionization parameter $(\xi)$ of the accretion disk, where $\xi=L / n R^{2}$, with $L$ as the ionizing luminosity, $n$ as the gas density, and $R$ as the distance to the ionizing source.

gemperature at the inner disk.

${ }^{\mathrm{h}}$ Normalization of dis kbb, given by $N_{\text {disk }}=\left(R_{\text {in }} / D_{10}\right)^{2} \cos \theta$, where $R_{\text {in }}$ is the apparent inner disk radius (in kilometers), $D_{10}$ is the distance to the source (in units of $10 \mathrm{kpc}$ ), and $\theta$ is the angle of inclination of the disk (in degrees; Kubota et al. 1998).

i Asymptotic power-law photon index.

$\mathrm{j}$ Electron temperature determining the high-energy rollover.

${ }^{\mathrm{k}}$ When the normalization of nthComp is unity, the corresponding model flux is 1 photon $\mathrm{keV}^{-1} \mathrm{~cm}^{-2} \mathrm{~s}^{-1}$ at $1 \mathrm{keV}$.

${ }^{1}$ Inner radius of the accretion disk.

${ }^{\mathrm{m}}$ The normalization of xillverCp $\left(N_{\mathrm{xil}}\right)$ is defined such that for an incident spectrum with flux $F_{\mathrm{X}}(E)$ incident on a disk with density $n_{\mathrm{e}}$ and with ionization

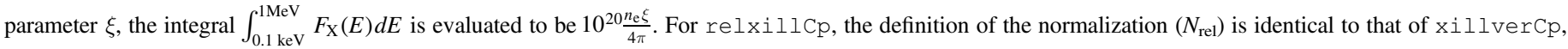
except that the flux reaching the observer is modified due to relativistic effects (see Dauser et al. 2016).

${ }^{\mathrm{n}}$ Unabsorbed flux (for reference, $1 \mathrm{Crab}=2.2 \times 10^{8} \mathrm{erg} \mathrm{cm}^{2} \mathrm{~s}^{-1}$ in the $2-10 \mathrm{keV}$ band).

${ }^{\mathrm{o}}$ Luminosity of the source in terms of its Eddington limit, computed assuming the mass of the black hole to be $M=10 M_{\odot}$, a distance of $D=8 \mathrm{kpc}$ (corresponding to $L_{\mathrm{Edd}}=1.25 \times 10^{38} \mathrm{erg} \mathrm{s}^{-1}$ ), and from the 0.01 to $100 \mathrm{keV}$ fluxes derived from the best-fit parameters.

${ }^{\mathrm{p}}$ Reflection strength, defined to be the ratio of the observed fluxes of the reflected component (relxillCp) and the incident component (nthComp) in the $20-40 \mathrm{keV}$ energy band.

${ }^{\mathrm{q}}$ Reduced $\chi^{2}$, defined as the ratio of the best-fit $\chi^{2}$ to the number of degrees of freedom $(\nu)$.

$\lesssim 4.4 R_{\text {ISCO }}\left(\lesssim 3.56 R_{\mathrm{g}}\right)$ by the time the spectral hardness reaches $\mathrm{HR} \simeq 0.7$ and remains close to ISCO all the way across the bright intermediate states, until the bright soft state $(\mathrm{HR} \simeq 0.1)$. Our results are incompatible with an inner radius placed at hundreds of $R_{\mathrm{g}}$, as has been reported elsewhere (Cabanac et al. 2009; Done \& Diaz Trigo 2010; Tamura et al. 2012; Plant et al. 2015).

As part of our analysis, we quantify the reflection strength $\left(R_{\mathrm{S}}\right)$ by computing the ratio of the observed fluxes of the reflected component (relxillcp) and the incident component (nthComp) in the $20-40 \mathrm{keV}$ energy band. The reflection strength is seen to be weakest $\left(R_{\mathrm{S}}=0.23_{-0.05}^{+0.05}\right)$ during the bright hard state $(\mathrm{HR} \simeq 0.8)$, and during the bright intermediate states $(0.7 \lesssim \mathrm{HR} \lesssim 0.2), R_{\mathrm{S}}$ is seen to oscillate between 0.21 and 0.47 . This is followed by a steep increase in its value, culminating at $R_{\mathrm{S}}=1.32_{-0.64}^{+0.37}$, by the time the system reaches the bright soft state (HR $\sim 0.1$ ). This is a 2.7 to $\sim 8.8$ fold rise in the reflection strength from its value during the inception of bright intermediate state transition.

\subsubsection{The 2004-2005 Outburst (Lower Flux)}

The 2004-2005 outburst (golden-brown color in Figure 2) is of particular interest because it exhibits a different hysteresis loop in the HID compared to the other outbursts. In this 
Table 3

Best-fit Parameters and the Corresponding 90\% Confidence Intervals Obtained from Fitting the RXTE/PCA Spectra of GX 339-4 from Its 2004-2005 Outburst Using the Model TBabs (diskbb+nthComp+relxillCp+xillverCp) for Different Hardness Ratio Values across the Bright Intermediate State (see Section 3.2.1)

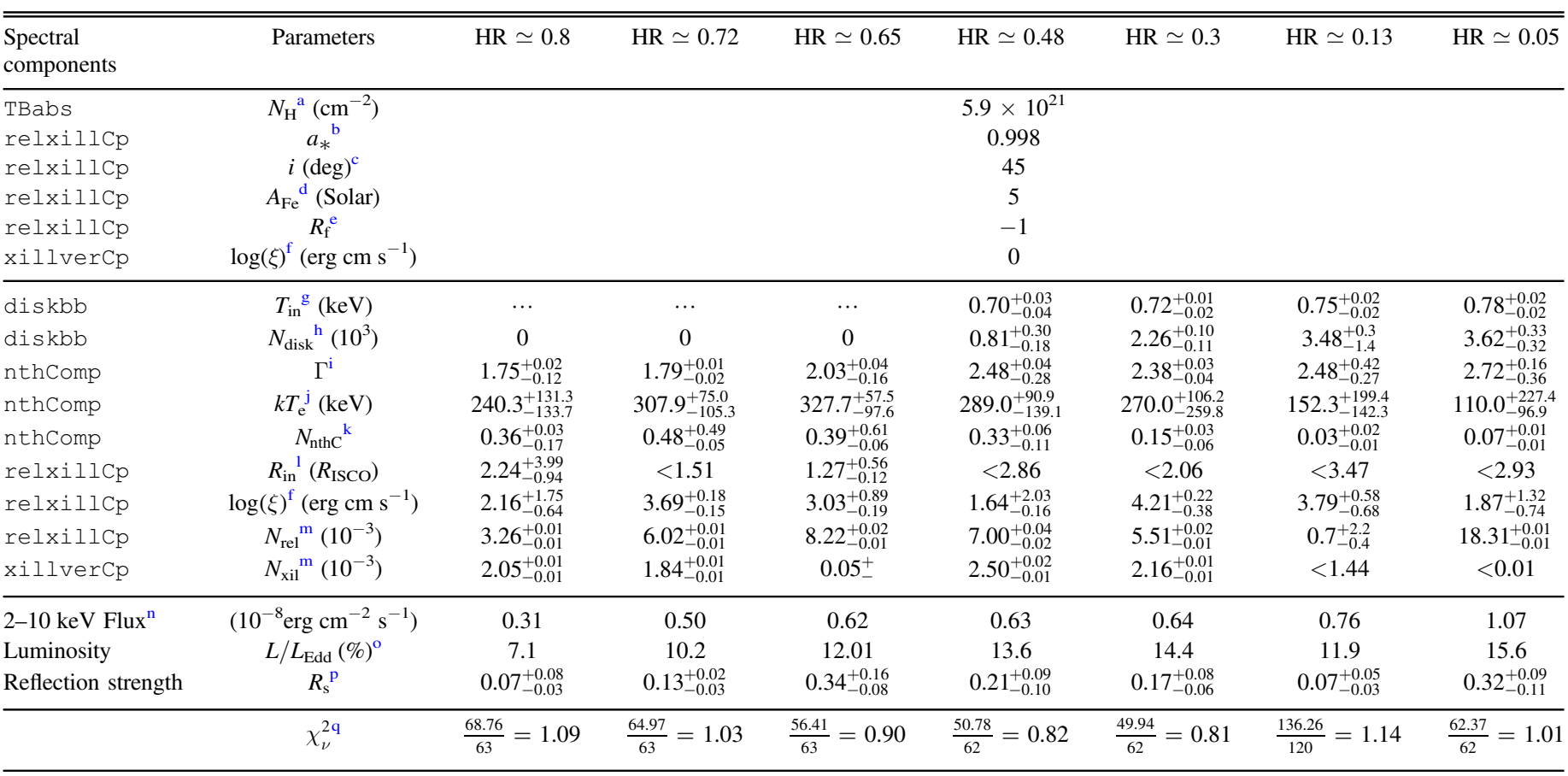

Notes.

${ }^{\mathrm{a}}$ Hydrogen column density.

${ }^{\mathrm{b}}$ Dimensionless spin parameter of the black hole $\left(a_{\star}=c J / G M^{2}\right.$, where $J$ is the angular momentum of the black hole).

${ }^{\mathrm{c}}$ Disk inclination.

${ }^{\mathrm{d}}$ Iron abundance of the material in the accretion disk.

${ }^{\mathrm{e}}$ Reflection fraction, defined as the ratio of the reflected flux to the flux in the power-law component, in the $20-40 \mathrm{keV}$ band.

${ }^{\mathrm{f}} \log$ of the ionization parameter $(\xi)$ of the accretion disk, where $\xi=L / n R^{2}$, with $L$ as the ionizing luminosity, $n$ as the gas density, and $R$ as the distance to the ionizing source.

gemperature at the inner disk.

${ }^{\mathrm{h}}$ Normalization of diskbb, given by $N_{\text {disk }}=\left(R_{\mathrm{in}} / D_{10}\right)^{2} \cos \theta$, where $R_{\text {in }}$ is the apparent inner disk radius (in $\mathrm{km}$ ), $D_{10}$ is the distance to the source (in units of 10 $\mathrm{kpc}$ ), and $\theta$ is the angle of inclination of the disk (in deg) (Kubota et al. 1998).

i Asymptotic power-law photon index.

$\mathrm{j}$ Electron temperature determining the high-energy rollover.

${ }^{\mathrm{k}}$ When the normalization of nthComp is unity, the corresponding model flux is 1 photon $\mathrm{keV}^{-1} \mathrm{~cm}^{-2} \mathrm{~s}^{-1}$ at $1 \mathrm{keV}$.

${ }^{1}$ Inner radius of the accretion disk.

${ }^{\mathrm{m}}$ Normalization of xillverCp $\left(N_{\mathrm{xil}}\right)$ is defined such that for an incident spectrum with flux $F_{\mathrm{X}}(E)$ incident on a disk with density $n_{\mathrm{e}}$, and with ionization parameter $\xi$, the integral $\int_{0.1 \mathrm{keV}}^{1 \mathrm{MeV}} F_{\mathrm{X}}(E) d E$ is evaluated to be $10^{20} \frac{n_{\mathrm{e}} \xi}{4 \pi}$. For relxillCp, the definition of the normalization $\left(N_{\text {rel }}\right)$ is identical to that of $x i l l v e r C p$, except that the flux reaching the observer is modified due to relativistic effects (see Dauser et al. 2016).

${ }^{\mathrm{n}}$ Unabsorbed flux (for reference, $1 \mathrm{Crab}=2.2 \times 10^{8} \mathrm{erg} \mathrm{cm}^{2} \mathrm{~s}^{-1}$ in $2-10 \mathrm{keV}$ band).

${ }^{\mathrm{o}}$ Luminosity of the source in terms of its Eddington limit, computed assuming the mass of the black hole to be $M=10 M_{\odot}$, a distance of $D=8 \mathrm{kpc}$ (corresponding to $L_{\mathrm{Edd}}=1.25 \times 10^{38} \mathrm{erg} \mathrm{s}^{-1}$ ), and from the 0.01 to $100 \mathrm{keV}$ fluxes derived from the best-fit parameters.

${ }^{\mathrm{p}}$ Reflection strength, defined to be the ratio of the observed fluxes of the reflected component (relxillCp) and the incident component (nthComp) in the $20-40 \mathrm{keV}$ energy band.

${ }^{\mathrm{q}}$ Reduced $\chi^{2}$, defined as the ratio of the best-fit $\chi^{2}$ to the number of degrees of freedom $(\nu)$.

outburst, the hard to intermediate state transition occurs at a luminosity that is two to three times lower than what is observed in the case of the other outbursts (see the luminosity row in Tables 2 and 3). The physical mechanisms that trigger the hysteresis observed in outbursts, and particularly the anomalous behavior of the 2004-2005 outburst, are not very well understood. This leaves us with the question of whether the global disk parameters including the inner disk radius differ in any way across the bright intermediate states of this outburst from the one previously analyzed (2002-2003).

For our exploratory analysis, we choose seven observations across the bright intermediate states of this outburst (see
Table 1). The overall model setup and the analysis procedure are identical to what is described in Section 3.2.1 for the 2002-2003 outburst. The best-fit parameters and the $90 \%$ confidence intervals obtained by fitting the 2004-2005 observations with our reflection model are listed in Table 3. The spectral data, total fitted model, the individual model components, and the fit residuals are plotted in Figure 4(b).

Very much like the 2002-2003 outburst, the harder spectra of the 2004-2005 outburst ( $\mathrm{HR} \simeq 0.8,0.72,0.65)$ do not exhibit the need for a blackbody component. Therefore, we freeze the normalization parameter of the diskbb component to 0 and fit for rest of the parameters. This restricts us from 

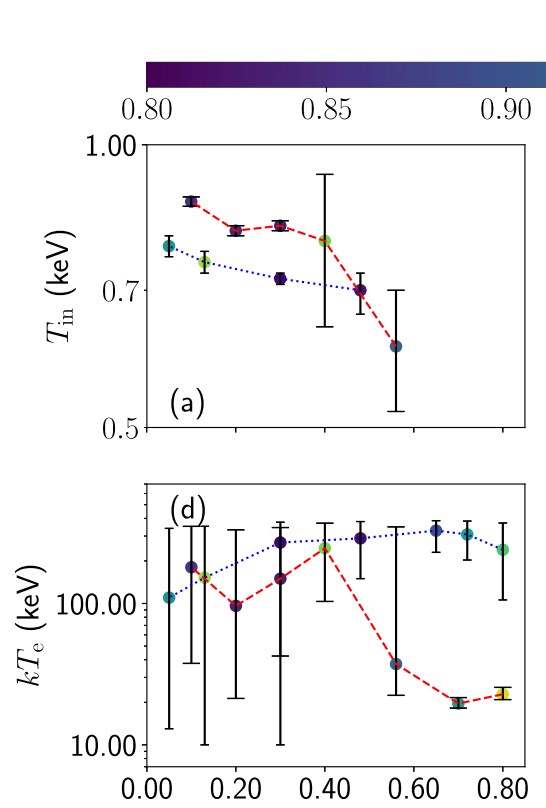
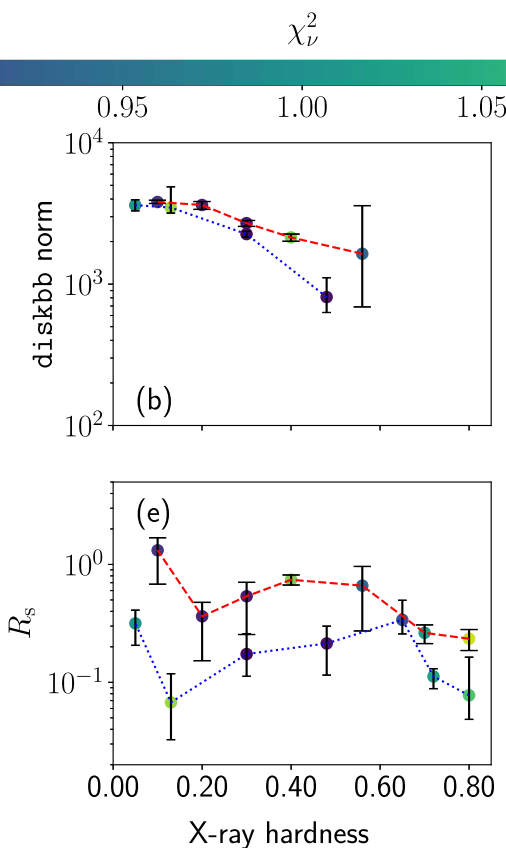

1.05
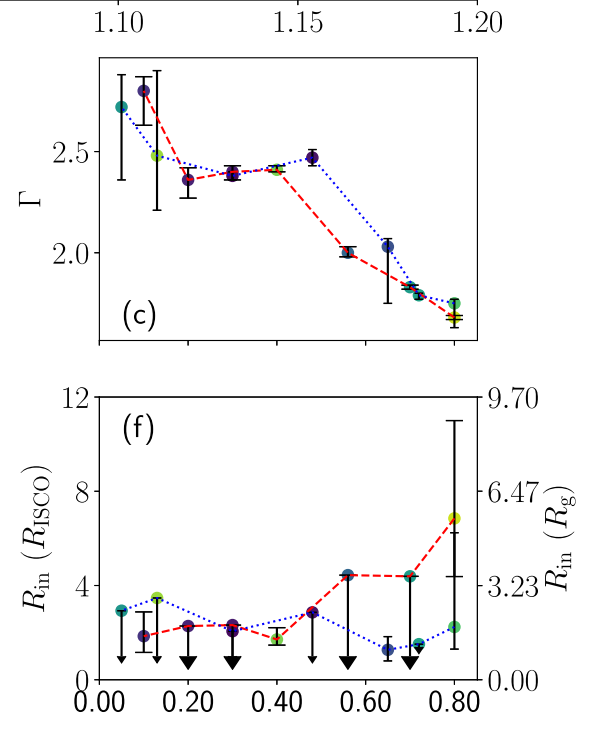

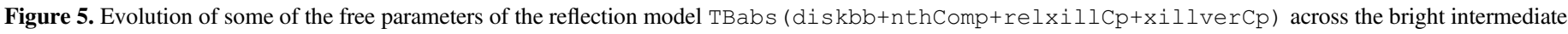

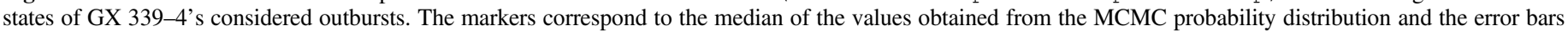

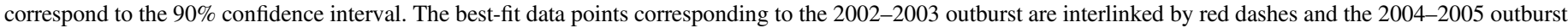

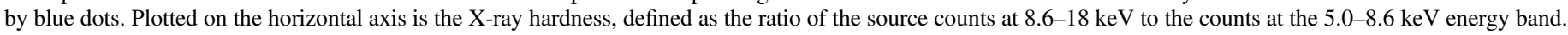

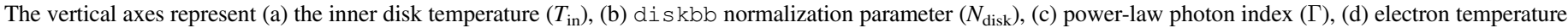

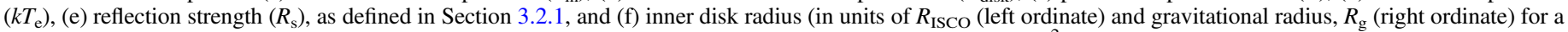

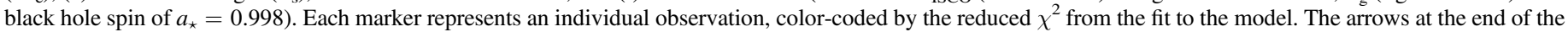
error bars indicate that the particular model parameter is unconstrained in that direction.

constraining the temperature of the inner disk for the hardest three observations. However, during the intermediate to soft states, the disk temperature of the 2004-2005 outburst is also consistently lower than the 2002-2003 outburst, which makes sense if the accretion rate $(\dot{M})$ is higher in the 2002-2003 outburst, although $\dot{M}$ need not be the only factor. We find that, except for the coronal electron temperature $\left(k T_{\mathrm{e}}\right)$, all the other parameters of the disk trace a similar trend across the bright intermediate states of the 2002-2003 outburst. Throughout this transition, $k T_{\mathrm{e}}$ values are seen to be unconstrained, with its lower limits found to be $>100-200 \mathrm{keV}$ during the hard to intermediate states $(\mathrm{HR} \simeq 0.8-0.48)$ and to be $>10 \mathrm{keV}$ during the softer states $(\mathrm{HR} \simeq 0.3-0.05)$.

Throughout the bright intermediate states of the 2004-2005 outburst, we find that the inner disk radius never recedes away by more than $6.23 R_{\mathrm{ISCO}}\left(5.04 R_{\mathrm{g}}\right.$ at $\left.\mathrm{HR} \sim 0.8\right)$, and the evolutionary trend at later times (HR $<0.8)$ is seen to be very much akin to the 2002-2003 outburst (see Figure 5).

For this outburst, the reflection strength is seen to be the weakest $\left(R_{\mathrm{s}}=0.07_{-0.03}^{+0.08}\right)$ during the bright hard state $(\mathrm{HR} \simeq 0.8)$, and during the bright intermediate states $(0.7 \lesssim \mathrm{HR} \lesssim 0.2), R_{\mathrm{s}}$ is seen to oscillate between 0.10 and 0.12 . This is followed by a steep rise in its value, culminating at $R_{\mathrm{S}}=0.32_{-0.11}^{+0.09}$, by the time the system reaches the bright soft state $(\mathrm{HR} \sim 0.1)$. This is a 2.1 to $\sim 8.1$-fold rise in the reflection strength from its value during the inception of bright intermediate state transition. This analysis demonstrates that although the reflection strength follows a similar evolutionary pattern across the bright intermediate states of the 2002-2003 and 2004-2005 outbursts, its magnitude is higher for the outburst with higher state transition luminosity (2002-2003) and lower for the outburst with lower state transition luminosity (2004-2005).

See Figure 5 for a visual comparison of the evolution of key parameters from the reflection spectrum analysis across the bright intermediate states of the 2002-2003 and 2004-2005 outbursts.

\subsubsection{MCMC Analysis}

In order to substantiate the parameter values derived from the complex reflection model, we perform a full-fledged MCMC statistical analysis for all 14 reflection fits (see Section 4 of Steiner \& McClintock 2012 for more details). The parameter space is explored in the MCMC analysis for each spectral fit with 50 walkers (distinct chains), which explore the parameter space in a sequence of 900,000 affine-invariant stretch-move steps (Foreman-Mackey et al. 2013). After having been initialized in a cluster distributed around the best-fit parameter values, the first $30 \%$ of the elements traversed by each walker are discarded as the "burn-in" phase, during which the chain reaches its stationary state. The autocorrelation length, which is the interval over which the chain forgets its previous location, for each walker is computed with respect to the lag in the length traversed. A typical value of the autocorrelation length is found to be $\sim 10,000$ elements $(\sim 10 \%)$, which corresponds to the net number of independent samples of the parameter space to be 90 per walker. All those walkers, whose autocorrelation values do not reach zero before it traverses $\sim 10 \%$ of the total chain length, are considered to be not converged and are discarded. From the full distribution, we obtain a probability distribution for any given set of parameters of interest by marginalizing over all the other variables that are outside that set. Logarithmically flat priors are adopted for all model components' normalization parameters. To regularize the space over which the parameters 
are sampled from a finite interval to a real line, each parameter is remapped using a logit transformation.

In Appendix $\mathrm{C}$, we show the contour maps and probability distributions for the set of the most relevant physical parameters, derived using the MCMC analysis. For the cases of $\mathrm{HR} \simeq 0.80$ and 0.70 in the 2002-2003 outburst, and $\mathrm{HR} \simeq 0.8,0.72$, and 0.65 in the 2004-2005 outburst, the diskbb norm parameter is not shown in the contour maps because the spectra are hard enough to require a disk blackbody component to describe it, and hence the parameter normalization is frozen to zero there (see Sections 3.2.1 and 3.2.2). For all the observations modeled with a free diskbb normalization parameter, the parameter space spanned by the walker clearly indicates a strong anticorrelation between $T_{\text {in }}$ and the component's norm, while $\Gamma$ and $R_{\text {in }}$ show little dependence on other parameters (see Figures 10 and 11). However, the hardstate observations do reveal a degeneracy in the value of the spectral index. The MCMC analysis performed here also helps us in revealing the bimodal distribution of the parameter values, seen in the contour plots as two different clusters of points $(\mathrm{HR} \simeq 0.8,0.65$, and 0.48 in Figure 11). Despite the fact that some parameters exhibit bimodal distributions, we note that they do not necessarily provide the best physical interpretation of the data and are just indicative of a few walkers being converged at local minima. The median value of each free parameter's distribution and the $90 \%$ confidence interval derived from the chains are listed in Tables 2 and 3.

\subsection{Fitting without the RELXILL Model}

Our analysis of the selected spectra from the bright intermediate states using the standard (blurred+unblurred) reflection model has indicated a relatively stable state of the inner edge of the accretion disk. Yet, continuum parameters like $T_{\text {in }}$, the diskbb norm, and $k T_{\mathrm{e}}$ are seen to be changing, in parallel with the softening of the spectra (see Tables 2 and 3). In this section, we perform an alternate analysis, which, although is not rigorous on the reflection component, can probe the continuum emission from the disk and corona to greater profundity. This analysis employs a selfconsistent Comptonized accretion disk model that also accounts for the scattering of the disk photons by the corona. Therefore, we note that the Comptonization continuum provided by this model is not identical to that of the previously employed reflection model, and we value this distinction. With this setup, our full model of the Comptonized accretion disk in this case is a convolution of the multitemperature disk blackbody (diskbb) component with an empirical simplcut ${ }^{6}$ model (Steiner et al. 2017), which convolves a Comptonization Green's function with a seed photon spectrum, thereby redirecting the photon from the seed distribution (described by diskbb) into a power law. In this model, we use a simple Gaussian component to account for the Fe $\mathrm{K}$ emission and the smedge component for the smeared absorption edge. The TBabs and the smedge components are handled the same way as mentioned in Section 3.1, and we fit for the rest of the free parameters of the model. Therefore, the final model setup in XSPEC notation is TBabs*smedge ( implcut $\otimes$ diskbb+Gaussian). We perform this analysis to retrieve meaningful coronal electron temperatures and hard-state $T_{\text {in }}$, which the previous reflection analysis could not yield a constraint on, and to independently estimate the inner disk radius from the diskbb norm.

\footnotetext{
6 http://jfsteiner.com/simplcut/
}

With this model, we fit a series of observations monotonically decreasing in their hardness from the bright hard to soft states of the 2002-2003 outburst. The relatively simple nature of this model allows us to include more observations from the bright soft state than the reflection analysis. The evolution of the key parameters of the disk and the corona, viz., $T_{\text {in }}$, diskbb norm, photon power-law index $(\Gamma)$, the fraction of seed photons scattered into power-law distribution $\left(f_{\mathrm{sc}}\right)$, optical depth $(\tau)$, and the coronal electron temperature $\left(k T_{\mathrm{e}}\right)$ is depicted in Figure 6. The optical depth is derived by assuming a covering fraction of unity using the relation $\tau=-\ln \left(1-f_{\mathrm{sc}}\right)$ (Steiner et al. 2017) and depicted along with the other parameters in Figure 6(f), for the sake of completion.

The increasing nature of $T_{\text {in }}$ with the softening of the spectra is seen to persist until the spectral hardness reaches a value of $\mathrm{HR} \simeq 0.3$, followed by a saturation of its value at $\sim 0.75 \mathrm{keV}$ until the bright soft state. On the other hand, the norm of the diskbb parameter, being degenerate with $T_{\text {in }}$, traces a decreasing trend as the spectra softens until the spectral hardness reaches a value of $\mathrm{HR} \simeq 0.3$. This trend is followed by a saturation of its value at $\sim 3 \times 10^{3}$ until the bright soft state. The power-law index, which is flattest during the hard state $(\Gamma \sim 1.6)$, reaches a value of $\Gamma \sim 2.4$ during the bright soft state, passing by the spectral hardness value of $\mathrm{HR} \simeq 0.3$, where it reaches its maximum value of $\Gamma \sim 2.7$. This value of the spectral hardness corresponds to what is known as the "SPL state" as defined by McClintock \& Remillard (2006) and first observed in this source by Miyamoto et al. (1991). Unlike the previously mentioned parameters, we do not observe any stark kink in the evolution of the scattered fraction $\left(f_{\mathrm{sc}}\right)$, and therefore $\tau$, at the SPL state. They exhibit a strong positive correlation with the spectral hardness throughout the bright intermediate states. The coronal electron temperature $\left(k T_{\mathrm{e}}\right)$ can be constrained with this model only during the bright hard to intermediate states $0.8<\mathrm{HR}<0.56$ (see Figure 6). The value of $k T_{\mathrm{e}}$ obtained from this analysis $(15 \sim 20 \mathrm{keV}$ in $0.8<\mathrm{HR}<0.56$, unconstrained elsewhere) closely resembles the value of $k T_{\mathrm{e}}$ obtained from the reflection analysis of the 2002-2003 outburst (see Table 2). Analysis of the reflection spectrum convolved with the self-consistently Comptonizing simplcut model is deferred to a future work.

\section{Discussion}

\subsection{Inner Disk Radius $\left(\mathrm{R}_{\text {in }}\right)$}

A number of studies (e.g., Done \& Diaz Trigo 2010; Basak \& Zdziarski 2016) have reported a broadening in the relativistic Fe $\mathrm{K}$ line as the spectra becomes softer and therefore have suggested that the inner radius correlates with the X-ray HR. Should this be the case, the signatures of the increase in line width with the softening of the spectra should also be seen with $R X T E /$ PCA. $^{7}$ The data/model ratio plot in Figure 3 shows the Fe $\mathrm{K}$ emission feature, plotted for three different representative values of HR across the bright intermediate states. Clearly, no significant broadening of the emission line can be seen, as the spectra softens, accompanied with the decrease in HR value from 0.8 to 0.1 . Should the inner disk's truncation be greatly decreasing as the system approaches the soft state from the bright hard state, we would observe, as a consequence, a steady broadening of the emission line due to gravitational redshift

\footnotetext{
7 See Appendix A for a test of the potential of $R X T E / \mathrm{PCA}$ to detect the narrow $\mathrm{Fe} \mathrm{K}$ emission line, corresponding to large disk truncation.
} 

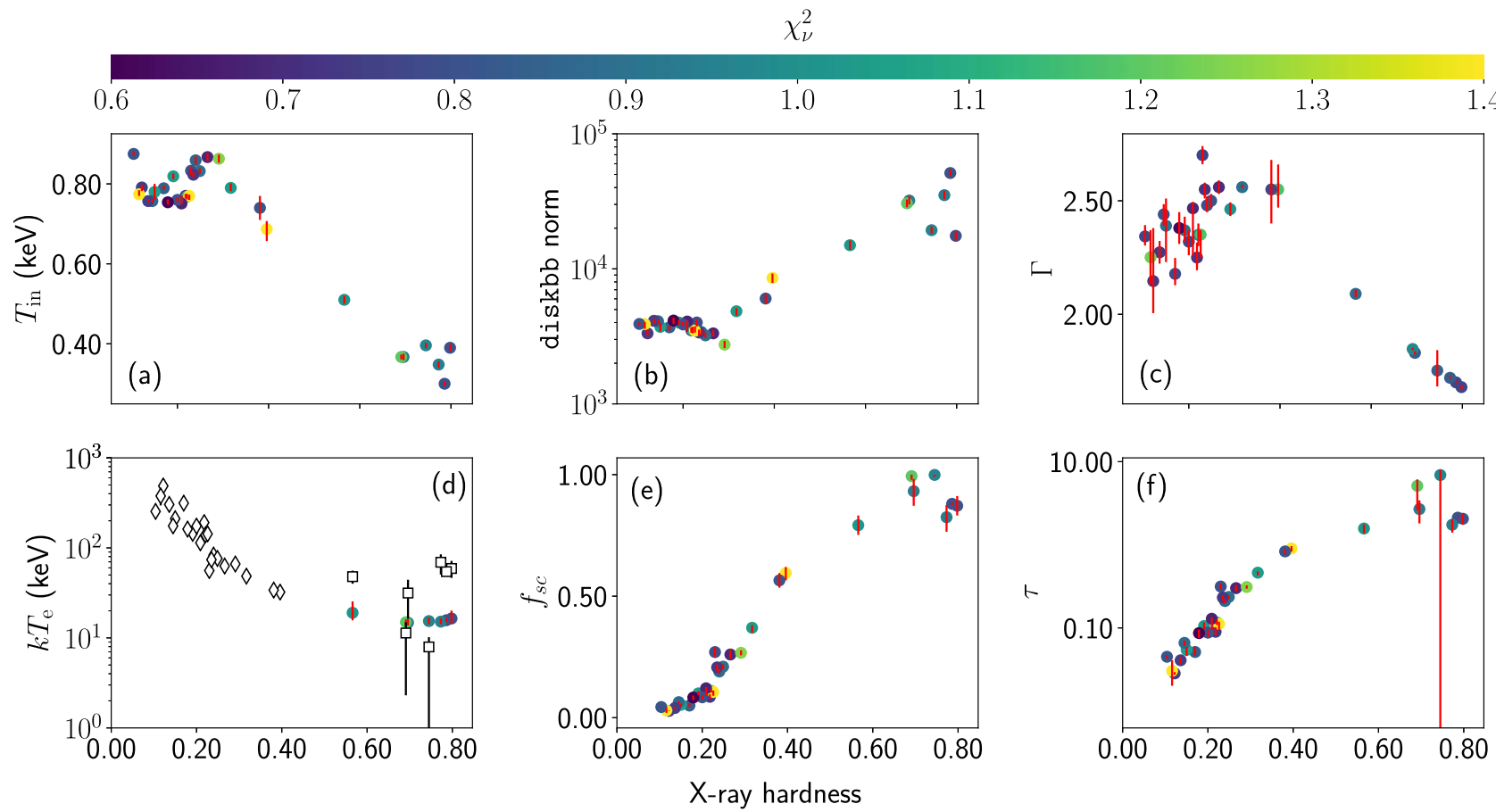

Figure 6. Evolution of the free parameters of the continuum model TBabs*smedge (simplcut $\otimes$ diskbb+Gaussian) across the bright intermediate states of the 2002-2003 outburst of GX 339-4. Plotted on the horizontal axis is the X-ray hardness, defined as the ratio of the source counts at 8.6-18 keV to the counts at 5.0-8.6 keV energy band. The vertical axes represent the (a) inner disk temperature $\left(T_{\text {in }}\right),(b)$ dis kbb norm, (c) power-law photon index $(\Gamma)$, (d) electron temperature - colored circles denote the values obtained from the best fit to the data (wherever constrained values are available), and open square and rhombus markers denote the analytically calculated values for the high and low optical depth regimes, respectively (see Section 4.4.1), (e) scattered fraction $\left(f_{\text {sc }}\right)$, and (f) optical depth $(\tau)$ for a covering fraction of unity. Each marker represents an individual observation, color-coded by the reduced $\chi^{2}$ from the fit to the model

(Ruszkowski \& Fabian 2000). Our result therefore qualitatively indicates that the inner radius of the accretion disk, which is presumed to be the source of the $\mathrm{Fe} \mathrm{K}$ emission line, is independent of the spectral hardness during the bright intermediate states' transition.

We then set out to quantitatively answer the question of where the inner disk truncates for the two outbursts we choose with different bright intermediate states' transition luminosities (2002-2003 and 2004-2005 outbursts). In order to answer this, we perform an analysis of the reflection spectrum using the RELXILL family of relativistic reflection models. Note that the diskbb norm $\left(N_{\text {disk }}\right)$ from the reflection fit is not indicative of the size of the disk, because it only models the unscattered (transmitted) disk emission. From the values of $R_{\text {in }}$ constrained from the analysis of the 2002-2003 and 2004-2005 outbursts (see Figure 5) using the relxillcp component, we find that the inner truncation of the accretion disk upon reaching very close to the ISCO by the onset of bright hard state (García et al. 2015, 2019; Wang-Ji et al. 2018) remains at a steady value $\left(R_{\text {in }} \sim R_{\mathrm{ISCO}}\right)$ regardless of the luminosity at which the black hole undergoes its transition across the bright hard to soft state. A plausible mechanism that can explain the sustained disk truncation close to the ISCO overcoming the putative magnetically arrested state of the disk (Narayan et al. 2003) is by annihilation of the accumulated central fields by quasi-periodic inversion of poloidal field lines (Igumenshchev 2009). According to Meyer-Hofmeister et al. (2009), the hard to soft state transition luminosity of an outburst occurs at a relatively lower luminosity (as in the 2004-2005 outburst) if the inner disk had remained stationary from the previous outburst (i.e., 2002-2003), plausibly due to recondensation of matter from the ADAF (Narayan et al. 1998). As per this picture, even though the inner edge of the outer disk could be highly truncated during quiescence and low/hard states, a weak inner disk might still be present during the low-luminosity states, giving rise to a small truncation radius as seen from our reflection study.

\subsection{Iron Abundance, Disk Density, and Jets}

A recent study of the high-density reflection models by Jiang et al. (2019) has indicated that the bright soft state spectra of GX 339-4 can be described by a near-solar iron abundance $\left(A_{\mathrm{Fe}}\right)$. We nevertheless use the aforementioned super-solar value, as the density of the disk at bright soft state is two orders of magnitude smaller than the low hard state. We note that the model flavor relxillD allows for a variable density of the disk. We, however, do not use it, as the current version of the model only allows for a fixed cutoff energy of $300 \mathrm{keV}$. That does not describe well the varying properties of the spectra seen across the bright intermediate states, and an appropriate model with a variable cutoff energy is not readily accessible.

There have been models of the formation and evolution of a synchrotron-emitting, large-scale transient jet during the bright intermediate state transition of GX 339-4 (Miyamoto \& Kitamoto 1991). In particular, the 2002-2003 outburst of this source began exhibiting large-scale jet during 2002 May 14 (Gallo et al. 2004). In our selected observations (Table 1), this is also the duration when the source makes a transition from $\mathrm{HR}=0.4 \rightarrow 0.3$ (SPL state). This transition, accompanied by a jet, is seen to give rise to several interesting signatures in the evolutionary trend of the source parameters. Such features include the inflections seen in the evolution of the power-law photon index (Figures 5(c) and 6(c)), reflection strength (Figure 5(f)), and the onset of saturation in the value of $T_{\text {in }}$ and diskbb norm (Figures 6(a) and (b)). Although it is perhaps easy 
to attribute the sudden changes seen in the source parameters to the emission of ballistic jets, the underlying physics of jet launching and its coupling with the disk/corona is still not well understood. However, from our results and previous discussion on the disk truncation (Section 4.1), we can at least argue that the advancement of the accretion disk toward the black hole need not be necessary to trigger the transient relativistic jet seen during the intermediate state transition in a microquasar like GX 339-4.

\subsection{Reflection Strength $\left(\mathrm{R}_{s}\right)$}

The approach we follow in order to parameterize the strength of the reflection is by calculating the ratio of the observed fluxes of the reflected component (relxillcp) to those of the incident component ( $\mathrm{nthComp}$ ) in the $20-40 \mathrm{keV}$ energy band, a definition that has been used by Keck et al. (2015) and Tao et al. (2015). This energy range is particularly of interest because, here, the reflection spectrum is independent of the ionization parameter $\xi$ (with no $\mathrm{Fe} \mathrm{K}$ fluorescent emission feature), and it also encompasses the peak of the Compton hump, where the reflection spectrum is dominated by Compton scattering. Another parameter quantifying reflection off the disk is the reflection fraction $\left(R_{\mathrm{f}}\right)$, which is defined as the ratio of the coronal intensity that illuminates the disk to the coronal intensity that reaches the observer. A correspondence between $R_{\mathrm{S}}$ and $R_{\mathrm{f}}$ for different disk inclination and black hole spin values is given in Dauser et al. (2016).

Our results indicate a weak reflection during the bright hard state of the 2002-2003 and 2004-2005 outbursts. A quick reasoning would be to ascertain that the disk is truncated far from the black hole, thus resulting in a diminished reflection from the disk. However, the estimates of the inner disk truncation from our reflection analysis (see Tables 2 and 3) and other works (García et al. 2015; Wang-Ji et al. 2018; García et al. 2019) indicate otherwise, and we would like to emphasize here that such a weak reflection does not necessarily imply a disk that is disrupted or truncated far from the black hole. Studies by Beloborodov (1999) and Malzac et al. (2001) have shown that a nonstatic corona during the hard state of a black hole can lead to an attenuated reflection strength, due to relativistic aberration. Another reason for this attenuation in $R_{\mathrm{s}}$ can be attributed to the large optical depth of the corona seen in the hard state (see Figure 6(f)), which dilutes the amplitude of the Fe line due to the resultant increased Compton scattering (Petrucci et al. 2001; Steiner et al. 2016). This attenuation in $R_{\mathrm{S}}$ during the bright hard state is followed by an increasing trend in the reflection strength with the softening of the spectra, which is consistent with the results of Steiner et al. (2016). This can be attributed to the "compactification" of the corona. In this paradigm, the ratio of the scale height of the corona $(h)$ to $R_{\text {in }}$ decreases with decreasing spectral hardness (Kara et al. 2019). Another possible scenario is when the radius of the corotating slab corona decreases, thereby leading to a softer emission (Zhang et al. 2019).

\subsection{Temperatures}

\subsubsection{Coronal Electron Temperature $\left(\mathrm{kT}_{e}\right)$}

The electron temperatures $\left(k T_{\mathrm{e}}\right)$ in the corona and its confidence intervals cannot be constrained well from our analysis, especially during the softer states of both the 2002-2003 and 2004-2005 outbursts. However, during the bright hard state, the $k T_{\mathrm{e}}$ values of the 2004-2005 outburst, having their lower limits at $\sim 100 \mathrm{keV}$, are higher than the upper limit of the $k T_{\mathrm{e}}$ values at the corresponding hardness value of the 2002-2003 outburst. This can be attributed to the inverse Compton cooling (IC) effect. The IC cooling power is given by

$$
P_{\mathrm{IC}}=\frac{4}{3} \sigma_{\mathrm{T}} c \beta^{2} \gamma^{2} U_{\mathrm{rad}},
$$

where $\sigma_{\mathrm{T}}$ is the Thomson cross section, $\beta$ is the velocity of the electrons in units of the speed of light $(c), \gamma$ is the Lorentz factor, and $U_{\text {rad }}$ is the energy density of the incident radiation. The bright intermediate states of the 2002-2003 outburst are on average $~ 3$ times more luminous than those of the 2004-2005 outburst, leading to a higher value of $U_{\text {rad }}$. This results in a more effective cooling of the corona in the 2002-2003 outburst compared to the 2004-2005 outburst. A reversal in the trend of $k T_{\mathrm{e}}$ is observed between the two outbursts, with the softening of spectra, as seen from Figure 5(d). However, we do not find this trend credible enough, owing to the unconstrained nature of the $k T_{\mathrm{e}}$ values during the softer states. We note here that the overall trend of the $k T_{\mathrm{e}}$ of the 2002-2003 outburst from reflection fitting is similar to that obtained from the nonreflection model (see Figures 5(d) and 6(d)).

In an isothermal corona, the pair/electron-ion plasma is known to Compton upscatter the thermal disk photons, thereby producing the observed power-law continuum. For known values of the power-law index $(\Gamma)$ and the electron temperature in the plasma $\left(k T_{\mathrm{e}}\right)$, the Thomson optical depth $(\tau)$ of the corona at different regimes can be estimated using the empirical relations (Pozdnyakov et al. 1983; Lightman \& Zdziarski 1987; Haardt \& Maraschi 1993):

$$
\begin{gathered}
\Gamma=-\frac{1}{2}+\sqrt{\frac{9}{4}+\frac{1}{\theta_{\mathrm{e}} \tau(1+\tau / 3)}}: \tau \gtrsim 1 \\
\Gamma=1+\frac{-\ln \tau+2 /\left(3+\theta_{\mathrm{e}}\right)}{\ln 12 \theta_{\mathrm{e}}^{2}+25 \theta}: \tau \lesssim 1,
\end{gathered}
$$

where $\theta_{\mathrm{e}}$ is a dimensionless temperature parameter given by $\theta_{\mathrm{e}}=k T_{\mathrm{e}} / m_{\mathrm{e}} c^{2}$, where $m_{\mathrm{e}} c^{2}=511 \mathrm{keV}$ is the rest mass energy of electron. The unconstrained nature of $k T_{\mathrm{e}}$ does not allow for the estimation of the optical depth, $\tau$ from Equations 2(a) and (b). However, the simplcut component of our model (see Section 3.3) provides us with the scattered fraction $\left(f_{\mathrm{sc}}\right)$, which is related to the optical depth $\tau$, by $\tau=-\ln \left(1-f_{\mathrm{sc}}\right)$, assuming a uniform density corona with a covering fraction of unity. The covering fraction of the corona for a realistic accretion disk is expected to be $<1$ (Wilkins \& Gallo 2015), and therefore our assumption places a lower limit on $\tau$. Using the analytical estimates of $\tau$ and the best-fit values of $\Gamma$, we retrieve back the electron temperatures using Equations 2(a) and (b) for different values of hardness across the bright intermediate states of the 2002-2003 outburst (see Figure 6(d)). The largest fraction of soft seed photons are produced in the softer state (see Figures 5(a) and 6(a)). Despite a large value of $U_{\text {rad }}$ during the softer states, the analytical estimate of $k T_{\mathrm{e}}$ is seen to grow higher, as the system gets softer. This is not unexpected, as the coronal electron temperature increases with a decrease in optical depth (lesser scattering), which is limited by the creation of $e^{ \pm}$pairs from magnetic reconnection events (Guilbert et al. 1983; Haardt \& Maraschi 1993; Beloborodov 2017).

We note here that all the coronal properties we place a constraint on are based on the assumption of seed photons being 
uniformly distributed and isotropic. For anisotropic cases, we refer readers to the recent results of Zhang et al. (2019).

\subsubsection{Inner Disk Temperature $\left(\mathrm{T}_{i n}\right)$ and the Spectral Hardening Factor $\left(\mathrm{f}_{\text {col }}\right)$}

The fact that GX 339-4 spends a couple of months in the bright intermediate states without exhibiting large changes in luminosity, but with just spectral hardness, also suggests a relatively stable configuration of the disk, which is plausible if the disk is near or at the ISCO. If this is the case, then the impending transition to the bright soft state should be triggered by a rather different physical process from any substantial radial motion of the disk. One plausible candidate is the surge in the accretion rate (Merloni et al. 2000; Narayan \& McClintock 2008; Schnittman et al. 2013). For a standard optically thick accretion disk, the blackbody temperature of the disk $\left(T_{\mathrm{bb}}\right)$ at a given radius $r$ is related to the accretion rate $\dot{M}$ through the relation

$$
\sigma T_{\mathrm{bb}}^{4}=\frac{G M}{r^{3}} \frac{3 \dot{M}}{8 \pi}\left[1-\left(\frac{r_{\mathrm{in}}}{r}\right)^{1 / 2}\right],
$$

where $M$ and $r_{\text {in }}$ denote the mass of the accreting body and the inner radius of the accretion disk. Equation (3) shows that for a relatively stable $r_{\text {in }}$, an increase in the accretion rate (as the spectra soften) should result in an increase in the inner disk temperature (possibly amplified by irradiation coupling; King \& Ritter 1998). We note here that our results manifest the same way (see Figure 6). In fact, the increasing trend of $T_{\text {in }}$ from our analysis with decreasing spectral hardness displays an agreement between the models with and without the reflection components (see Figures 5 and 6).

The inner disk temperature $\left(T_{\mathrm{in}}\right)$ returned by the diskbb component is the color temperature, which is related to the effective temperature ( $\left.T_{\text {eff }}\right)$ of the disk by $T_{\text {in }}=f_{\text {col }} T_{\text {eff }}$, where $f_{\text {col }}$ is the color-correction (a.k.a. spectral hardening) factor (Shimura \& Takahara 1995). Salvesen et al. (2013) had demonstrated that physically reasonable changes in the phenomenological color-correction factor $\left(f_{\text {col }}\right)$ can also provide a plausible description for the hard to soft state transition, without needing to invoke the radial motion of the inner accretion disk (Davis \& El-Abd 2019). Assuming a canonical value of $f_{\text {col }}=1.7$ at the bright soft state, we estimate the temperature-dependent values of $f_{\text {col }}$ for varying spectral hardnesses, using the scaling relation $f_{\text {col }} \propto T_{\text {in }}^{1 / 3}$ (Davis et al. 2006). The $T_{\text {in }}$ values used in this calculation are extracted from the model TBabs*smedge (simplcut $\otimes$ diskbb+Gaus sian). We use the values from this model in order to arrive at $R_{\text {in }}$ measurements independent of the results from the reflection model. Note that the dis kbb norm from the reflection fit is not indicative of the size of the disk, as it only models the unscattered (transmitted) disk emission. This is not the case with the diskbb norm from this alternate analysis involving the simplcut model. The normalization of the diskbb component is given by $N=\left(r_{\text {in }}^{\text {app }} / D_{10}\right)^{2} \cos \theta$, where $r_{\text {in }}^{\text {app }}$ is the "apparent inner disk radius," $D_{10}$ is the distance to the black hole in units of $10 \mathrm{kpc}$, and $\theta$ is the inclination value of the disk. Using the relationship between the apparent and the true inner disk radius (Kubota et al. 1998), the diskbb normalization $(N)$ can be rewritten in terms of $f_{\text {col }}$ as

$$
N=\left(\frac{r_{\text {in }}}{\Xi f_{\text {col }}^{2} D_{10}}\right)^{2} \cos \theta,
$$

where $r_{\text {in }}$ is the true inner disk radius (in units of $\mathrm{km}$ ) and $\Xi$ is the relativistic color-correction factor (of the order of unity). We would like to emphasize here that a negligible value of the diskbb normalization or a nonrequirement of the disk quasiblackbody component in the spectrum (as seen in the hard-state spectra of our reflection analysis) does not necessarily imply that the accretion disk is highly truncated, or even absent. A likely interpretation for this is that the cool thermal photons emitted by the disk are almost entirely Comptonized in a $\tau \gtrsim 1$ corona (see Figure 6(f)), and so the transmitted (unscattered) portion of that emission is weak. Note that this does not prevent one from being able to place a one-sided constraint of diskbb parameters with the simplcut model. Moreover, $R X T E / P C A$ has its low-energy cutoff at $\sim 3 \mathrm{keV}$, allowing only the Wien tail of the blackbody spectrum in the observable band. The blackbody component during the hard state of GX 339-4, primarily dominant at energies below PCA's energy sensitivity, has been observed with other instruments (Miller et al. 2006; Reis et al. 2008; Tomsick et al. 2008; Plant et al. 2015; Basak \& Zdziarski 2016).

As a next step, we plug in previously estimated values of $f_{\text {col }}$ from the scaling relation into Equation (4) and retrieve the evolution of the inner disk radius assuming the following about the system's intrinsic parameters: $M=8 \pm 2 M_{\odot}$, $D_{10}=0.8 \pm 0.2$, inclination $\theta=45^{\circ} \pm 15^{\circ}$, and the dimensionless spin parameter to be $a_{\star}=0.950 \pm 0.049$ (see Section 1). Figure 7 shows the evolution of the inner disk radius independently measured from the continuum fitting method, and the corresponding value of $f_{\text {col }}$ used at different hardness values.

From Figure 7, we see that the radius of the inner disk's truncation remains at a value of $\sim 2 R_{\text {ISCO }}$ across the bright intermediate states, thus independently confirming our results from the reflection analysis. We note here that changes seen in the norm of the diskbb component (as in Figure 6(b)) do not necessarily imply a change in the inner disk radius, due to the existing degeneracy between $f_{\text {col }}$ and $R_{\text {in }}$. On the other hand, previous works by Reynolds \& Miller (2013) and Salvesen et al. (2013) have demonstrated that order of magnitude changes seen in the diskbb normalization can be explained by merely invoking changes in the spectral hardening factor. Furthermore, with the steady increase in $f_{\text {col }}$ seen with the softening of the spectra, it is easier to conceive that the bright hard to soft state transition is perhaps triggered by the magnetic or surface properties of the accretion disk rather than by the sudden appearance of the disk. In particular, the strong toroidal magnetic fields generated by the magnetorotational instability (Velikhov 1959; Chandrasekhar 1960; Balbus \& Hawley 1991) would cause the disk to thicken, consequently decreasing the viscous inflow time (Begelman \& Pringle 2007; Johansen \& Levin 2008). The existence of a strong correlation between $f_{\text {col }}$ and the viscosity parameter ( $\alpha$; Shakura $\&$ Sunyaev 1973) in the inner regions of the disk is studied in Shimura \& Takahara (1995). This dependence can be explained on the grounds of a gradual increase seen in the electron temperature as the disk becomes optically thin (see Figure 6(f)), with an increase in $\alpha$ due to changes in $\dot{M}$. We refer readers to the works of Davis et al. (2005, 2006) and Davis \& El-Abd (2019) for a more comprehensive discussion on the dependence of $f_{\text {col }}$ on other physical processes. 


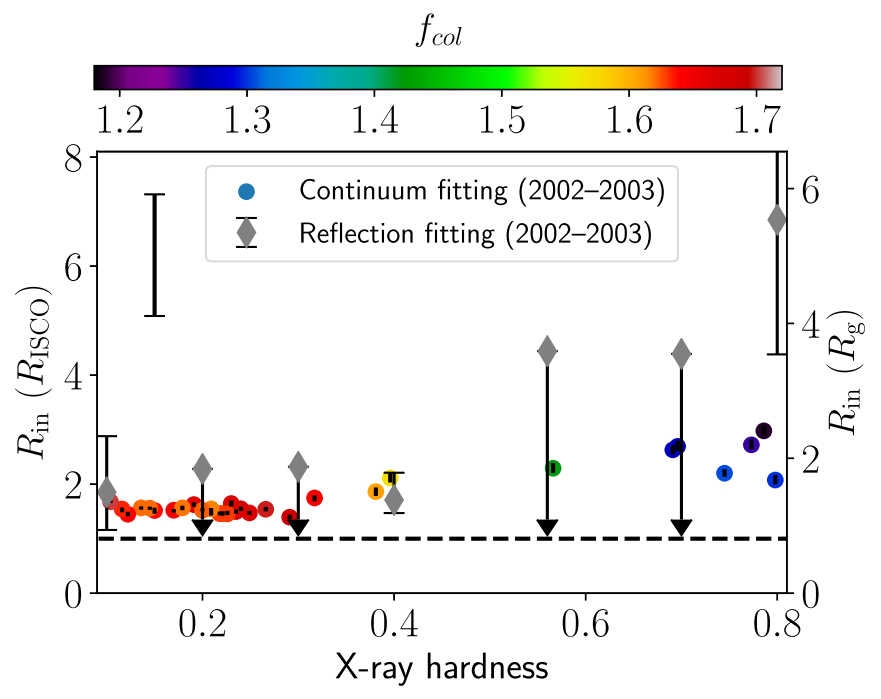

Figure 7. Independent measurements of the evolution of the inner disk radius $\left(R_{\text {in }}\right)$ from the continuum fitting method (round markers), in comparison with the results from the reflection method (gray rhombus markers), plotted against the X-ray hardness. The inner disk radii are plotted in units of $R_{\mathrm{ISCO}}$ (left ordinate) and gravitational radius ( $R_{\mathrm{g}}$; right ordinate), for a black hole spin of $a_{\star}=0.998$. The vertical error bars (on the marker) represent the propagated statistical uncertainties from the model fitting, and the systematic uncertainty arising from ignoring the system's intrinsic parameters is represented by the large vertical error bar with a cap at the top-left region of the figure. The round markers are color-coded with the value of the spectral hardening factor $\left(f_{\mathrm{col}}\right)$, which is used to calculate $R_{\text {in }}$.

\section{Summary}

In this paper, we perform an analysis of the reflection spectrum of the BHXB GX 339-4, as observed by RXTE during the transition from the bright hard to soft states of the 2002-2003 and 2004-2005 outbursts. We also perform an analysis of the continuum spectra from the bright intermediate state transition by employing a self-consistent Comptonized accretion disk model accounting for the scattering of the disk photons by the corona to arrive at the disk and coronal properties independent of the reflection modeling, and compare one against each other. Our chief results are the following:

1. Strong features of broad Fe K emission are seen in the data across the bright intermediate state transition, and the profile of the $\mathrm{Fe} \mathrm{K}$ emission is mostly insensitive to the Comptonization model used (Section 3.1).

2. Qualitatively, the width of the Fe $\mathrm{K}$ emission feature is seen to be nearly constant across the bright hard to soft state transition, indicating a quasi-static inner disk truncation radius (Section 3.1).

3. By fitting the data with the RELXILL family of relativistic reflection models, we conclude that the inner edge of the accretion disk reaches $\sim 9 R_{\mathrm{g}}$ by the onset of the bright hard state, and the truncation remains at $\lesssim 3 R_{\mathrm{g}}$ across the bright intermediate state transition (Section 3.2.1).

4. Regardless of the luminosity at which the source undergoes the bright hard to soft state transition in this source (as in the lower luminosity 2004-2005 outburst), the inner disk truncation is seen to be behaving similarly, as mentioned above (Section 3.2.2).

5. While disk and coronal parameters like the disk blackbody normalization, power-law photon index $(\Gamma)$, and $R_{\text {in }}$ are seen to be similar between the bright intermediate state transition of different luminosities, other parameters like $T_{\mathrm{in}}, k T_{\mathrm{e}}$, and reflection strength $\left(R_{\mathrm{s}}\right)$ are seen to be sensitive to the luminosity of state transition (Sections 3.2.1 and 3.2.2).

6 . In addition to reflection modeling, we also perform a continuum fitting analysis of several spectra across the bright intermediate transition of the 2002-2003 outburst by employing a self-consistent Comptonized accretion disk model accounting for the scatter of disk photons by the corona (Section 3.3).

7. With the measured values of the coronal optical depth and $\Gamma$ from the fitting procedure, we solve Equations 2(a) and (b) to find a strong anticorrelation of spectral hardness with $k T_{\mathrm{e}}$. With known values of the diskbb norm and estimates of the spectral hardening factor $\left(f_{\text {col }}\right)$, we solve Equation (4) to determine $R_{\text {in }}$ and independently find it corroborating with our results from reflection fitting (Section 4.4.2).

8. With the inner disk not seen to be physically moving toward the black hole significantly during the bright hard to soft state transition, the changes seen in the model parameters can be attributed to processes like increase in spectral hardening factor, compactification of the corona, collapse of the magnetically supported disk from being geometrically thick to thin, and increase in $\dot{M}$, among others. The question of the physical mechanism triggering the state transitions still remains uncertain (Section 4).

N.S. acknowledges the support from DST-INSPIRE, Caltech SURF, and Columbia University Dean's fellowships. J.A.G. acknowledges the support from NASA grant 80NSSC19K1020 and from the Alexander von Humboldt Foundation. R.M.T.C. has been supported by NASA ADAP grant 80NSSC17K0515. V.G. is supported through the Margarete von Wrangell fellowship of the ESF and the Ministry of Science, Research and the Arts Baden-Württemberg. We also thank Ronald A. Remillard for his contribution toward processing the data, which have been used in our analysis. This work was partially supported under NASA contract No. NNG08FD60C.

Facility: RXTE (PCA).

Software: XSPEC v12.9.1q (Arnaud 1996), RELXILL v1.4 (Dauser et al. 2014; García et al. 2014a), XILLVER (García \& Kallman 2010; García et al. 2013), PCACORR (García et al. 2014b).

\section{Appendix A \\ Potential of RXTE/PCA and RELXILL}

In this section, we test the capability of $R X T E / P C A$ and RELXILL in detecting large values of $R_{\text {in }}$ for an axisymmetric, stationary source radiating isotropically. ${ }^{8}$ Determining the capability of $R X T E /$ PCA and RELXILL to detect such narrow features is essential to conclude whether or not the inner disk is actually truncated at $R_{\text {in }} \gg R_{\mathrm{ISCO}}$. For this exercise, we generate synthetic spectra using the fakeit routine in XSPEC V12.9.1Q (Arnaud 1996). The simulated spectra are created with the response of $R X T E / P C A$ in the $\sim 3-45 \mathrm{keV}$ energy band with long-enough exposure such that they have at least a million counts in the considered energy range.

Using these specifications, 1000 spectra are synthesized with the model TBabs*relxill, with varying $R_{\text {in }}$ values for two cases: (i) $R_{\mathrm{f}}=0.2, a_{*}=0.998$ and (ii) $R_{\mathrm{f}}=0.7, a_{*}=0.2$, where $R_{\mathrm{f}}$ is the reflection fraction and $a_{*}$ is the dimensionless

\footnotetext{
8 A similar analysis with a different set of parameters of RELXILL has been performed by Choudhury et al. (2017).
} 


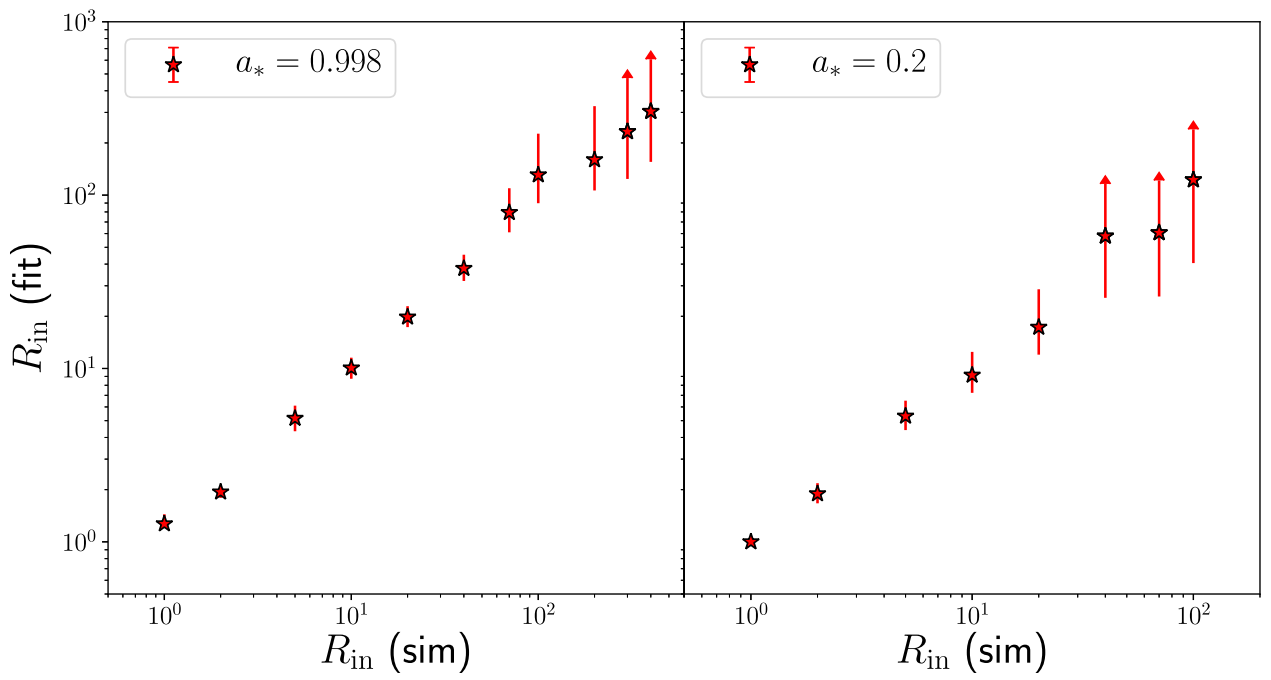

Figure 8. Best-fit $R_{\text {in }}$ values (vertical axes; in units of $R_{\mathrm{ISCO}}$ ) plotted against the $R_{\text {in }}$ used to simulate the spectra (horizontal axes). The error bars represent $90 \%$ confidence intervals. For $a_{\star}=0.998$, the fit goes unconstrained for a value of $R_{\text {in }} \gtrsim 120 R_{\text {ISCO }}$. On the other hand, for a small spin parameter of $a_{\star} \sim 0.2$, the fit goes unconstrained for an $R_{\text {in }}$ value as low as $\sim 40 R_{\mathrm{ISCO}}$. The parameters fed in to simulate the spectra in the left and right panels are described in Appendix A.

spin parameter. In both cases, the disk emissivity is fixed to the canonical value of 3 (Fabian et al. 1989), inclination to $45^{\circ}$, photon index $\Gamma$ to $2, \log (\xi)$ to 3 , and the iron abundance $A_{\mathrm{Fe}}$ to 5 ( $\times$ solar). The input values are chosen such that they represent the typical physical conditions present in most observed systems (García et al. 2013). The simulated spectra are then fitted with the model TBabs*relxill, where all the parameters are frozen to simulated values with $R_{\text {in }}$ as a free parameter. For cases (i) and (ii), $R_{\text {in }}$ cannot be constrained for values $\gtrsim 120 R_{\mathrm{ISCO}}$ and $\gtrsim 40 R_{\mathrm{ISCO}}$, respectively (Figure 8 ). This result shows that for a high-spin black hole source like that of GX 339-4, the data of RXTE/PCA with the RELXILL model are capable of constraining the inner radius of the accretion disk as large as $120 R_{\mathrm{ISCO}}$.

\section{Appendix B \\ Disparity with the Truncated Disk Model}

Having demonstrated the capability of RXTE/PCA and RELXILL in detecting a large disk truncation radius (Appendix A), we now simulate spectra corresponding to large disk truncation with the PCA instrument response. We use the best-fit model parameters (Model 2(i)) of Basak \& Zdziarski (2016) to simulate the spectra of their observations 3 and 7 using PCA's response. The parameter values used for simulating the spectra are listed in Table 4 . Basak \& Zdziarski (2016) had analyzed seven observations of GX 339-4 in its hard state, as observed by the XMM-Newton EPIC-pn detector. For our exercise, we choose the timing mode observations 3 and 7 from their list because of their high count rates and the "timing mode" of the observation. In addition, we simulate another spectrum with all the same parameters from fitting their observation 7, but with $R_{\text {in }}=R_{\mathrm{ISCO}}$, for comparison purpose.

In order to visualize the $\mathrm{Fe} \mathrm{K}$ reflection feature, we fit the simulated spectra with a simple absorbed power-law model and follow the procedures outlined in Section 3.1. Broadening of the Fe $\mathrm{K}$ line with decreasing $R_{\text {in }}$ values is seen in Figure 9, as expected. Note that observations 7 and 3 correspond to the bright softest and hardest state, respectively, in the sample of Basak \& Zdziarski (2016). Should the disk indeed be truncated at $\sim 100 R_{\mathrm{ISCO}}$ during any of our selected bright hard to soft

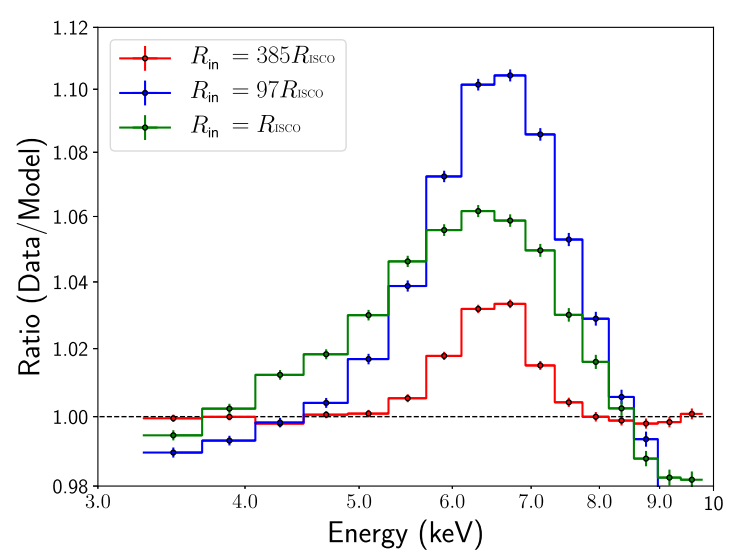

Figure 9. Data/model ratio plot produced by fitting the simulated spectrum from Basak \& Zdziarski (2016; see Table 4) using PCA's response with the absorbed power law model. Red and blue profiles correspond to their observations 3 and 7 , respectively. The green profile is simulated for the same set of parameters except for the disk truncation fixed at $R_{\mathrm{ISCO}}$, for comparison purposes.

Table 4

Parameter Values from Fitting the Best-fit Model 2(i) of Basak \& Zdziarski (2016) to the Data of Their Observations 3 and 7, Used for Simulating the Spectra with PCA's Response

\begin{tabular}{|c|c|c|c|}
\hline Component & Parameter & Obs. 3 & Obs. 7 \\
\hline TBabs & $N_{\mathrm{H}}\left(\mathrm{cm}^{-2}\right)$ & \multicolumn{2}{|c|}{0.71} \\
\hline nthComp & $k T_{\mathrm{e}}(\mathrm{keV})$ & \multicolumn{2}{|c|}{100} \\
\hline nthComp & $i(\mathrm{deg})$ & \multicolumn{2}{|c|}{43.8} \\
\hline relxill & $A_{\mathrm{Fe}}($ solar $)$ & \multicolumn{2}{|c|}{1} \\
\hline relxill & $a_{*}$ & \multicolumn{2}{|c|}{0.998} \\
\hline relxill & $E_{\text {cut }}(\mathrm{keV})$ & \multicolumn{2}{|c|}{150} \\
\hline relxill & $N_{\text {rel }}$ & \multicolumn{2}{|c|}{0.04} \\
\hline diskbb & $T_{\text {in }}(\mathrm{keV})$ & 0.19 & 0.21 \\
\hline diskbb & $N_{\text {disk }}$ & $7.74 \times 10^{5}$ & $4.95 \times 10^{4}$ \\
\hline nthComp & $\Gamma$ & 1.56 & 1.69 \\
\hline nthComp & $N_{\text {nthC }}$ & 0.19 & 0.52 \\
\hline relxill & $R_{\mathrm{in}}\left(R_{\mathrm{ISCO}}\right)$ & 385 & 97 \\
\hline relxill & $\log (\xi)\left(\mathrm{erg} \mathrm{cm} \mathrm{s}^{-1}\right)$ & 2.69 & 3.31 \\
\hline
\end{tabular}

Note. A short description of each parameter is provided under Table 2 . 
state observations, we would expect to see broadened emission feature as seen in Figure 9. However, our result (see Figure 3) does not exhibit any line broadening, indicative of a stable inner disk radius during the bright hard to soft state transition spanning across a wide range of hardness values. As discussed in Miller et al. (2010), this disparity can be attributed to the deteriorating effects of high photon pile-up in the timing mode observations of XMM-Newton. If not corrected for the pile-up effects, it can artificially make the continuum softer, resulting in a narrower $\mathrm{Fe} \mathrm{K}$ profile, thereby leading to estimates of truncation much larger than reality.

\section{Appendix C MCMC Parameter Probability Distributions}

In this section, we show the contour maps and probability distributions for the set of the most relevant physical parameters derived using the MCMC analysis (Figures 10 and 11). These contour maps demonstrate how well each parameter is constrained in the fit, and the level of correlation and degeneracy between parameters. For each map, we also show the 0.16, 0.5, and 0.84 quantiles. Refer to Section 3.2.3 for a detailed description of the MCMC setup and the results.

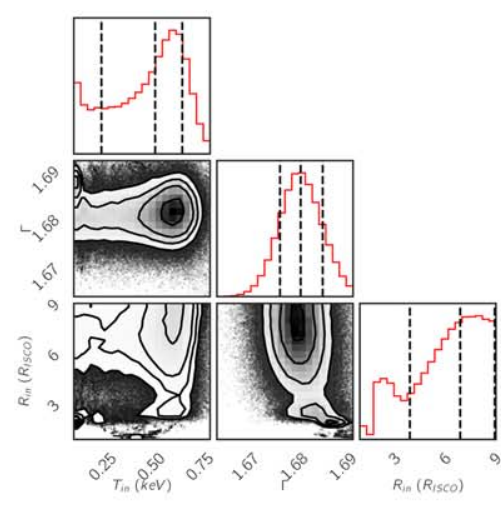

(a) $H R \simeq 0.80$

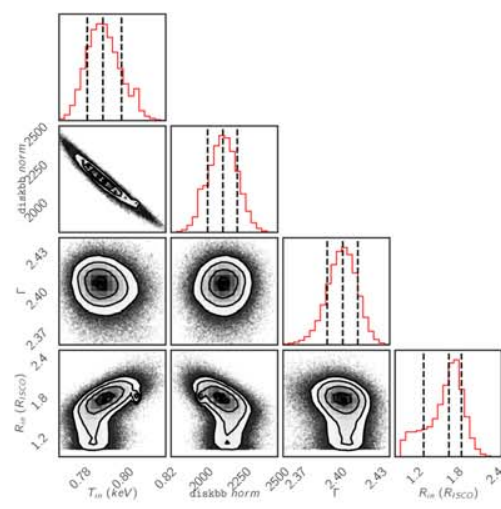

(d) $H R \simeq 0.40$

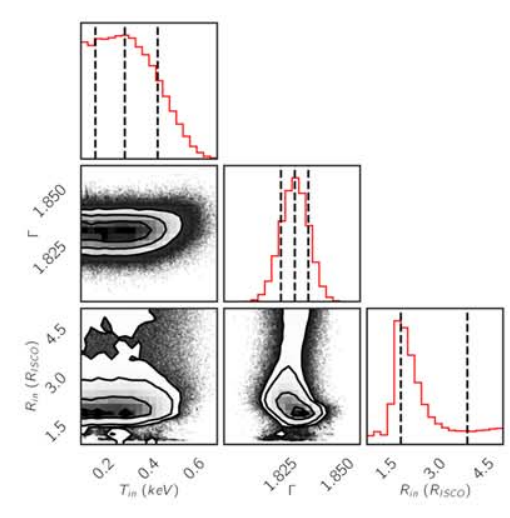

(b) $H R \simeq 0.70$

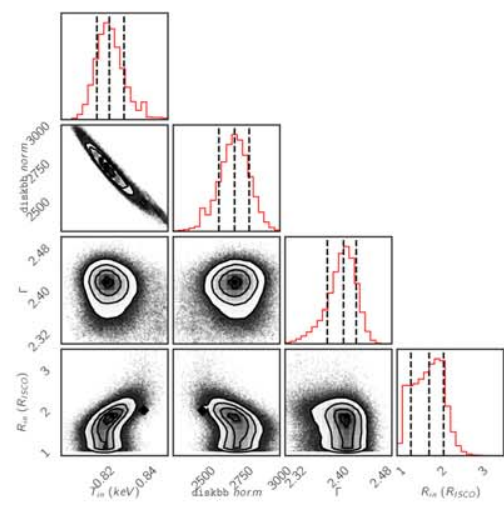

(e) $H R \simeq 0.30$

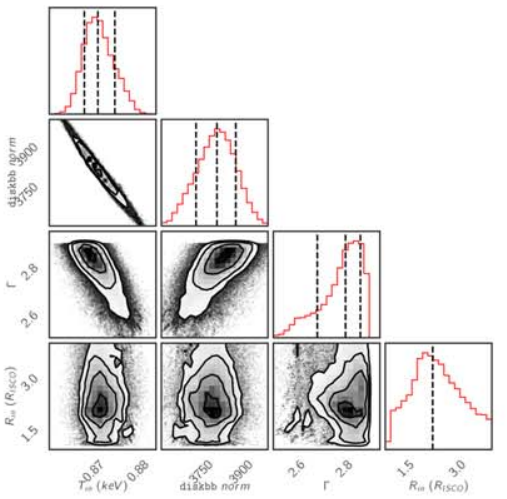

(g) $H R \simeq 0.10$

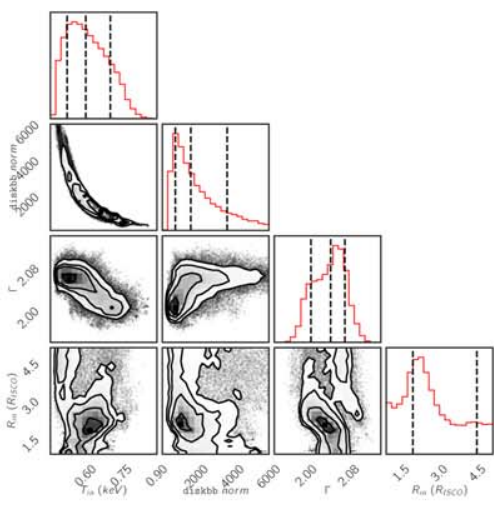

(c) $H R \simeq 0.56$

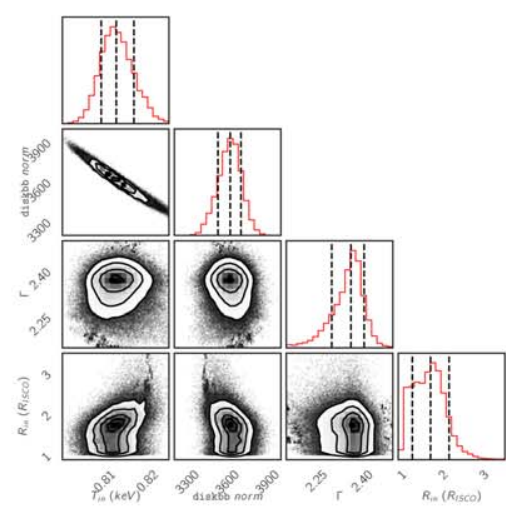

(f) $H R \simeq 0.20$

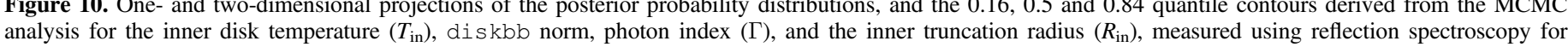

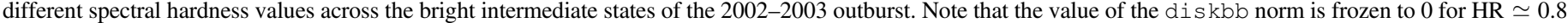
and $\mathrm{HR} \simeq 0.7$. These figures are produced using the corner package (Foreman-Mackey 2016). 


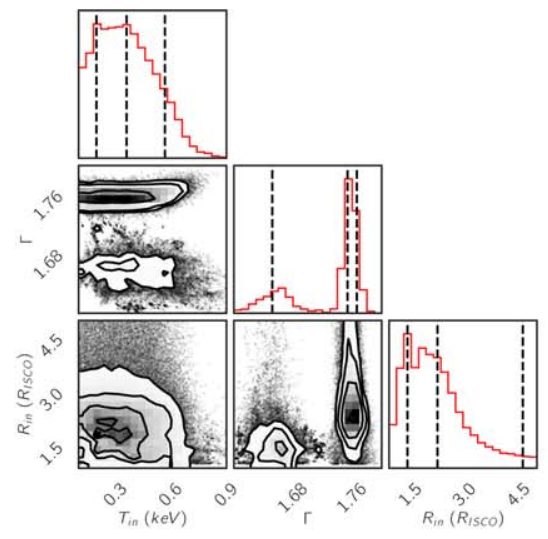

(a) $H R \simeq 0.80$

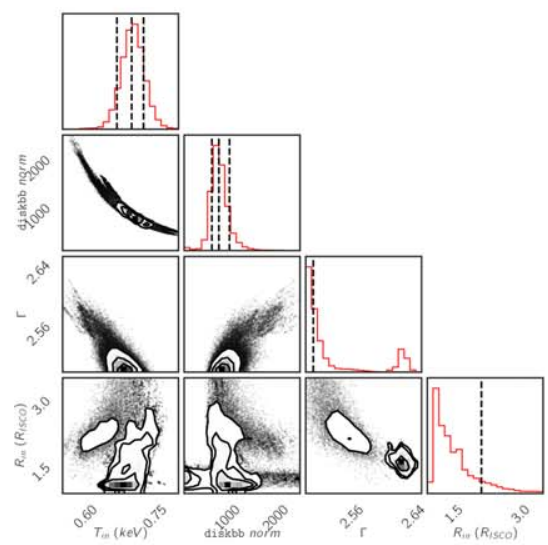

(d) $H R \simeq 0.48$

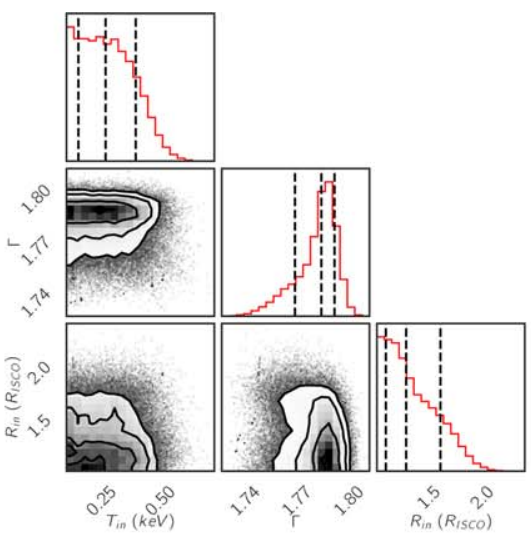

(b) $H R \simeq 0.72$

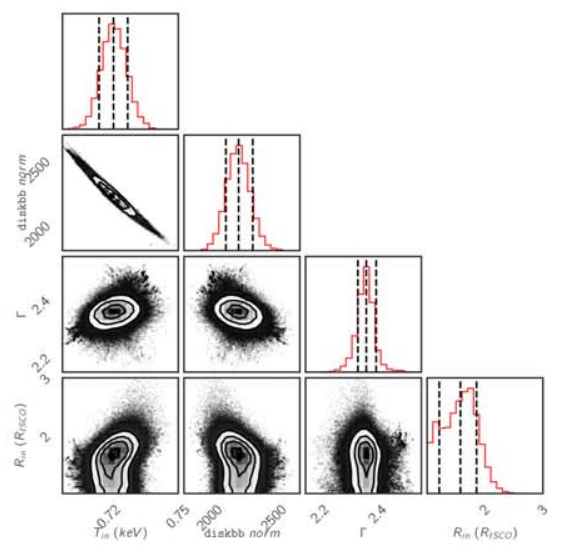

(e) $H R \simeq 0.30$

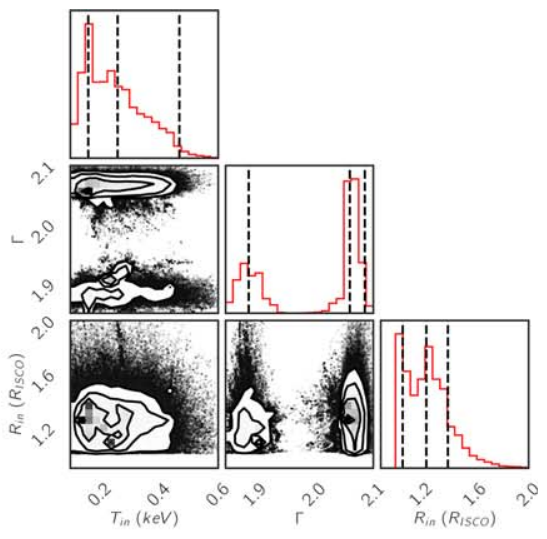

(c) $H R \simeq 0.65$

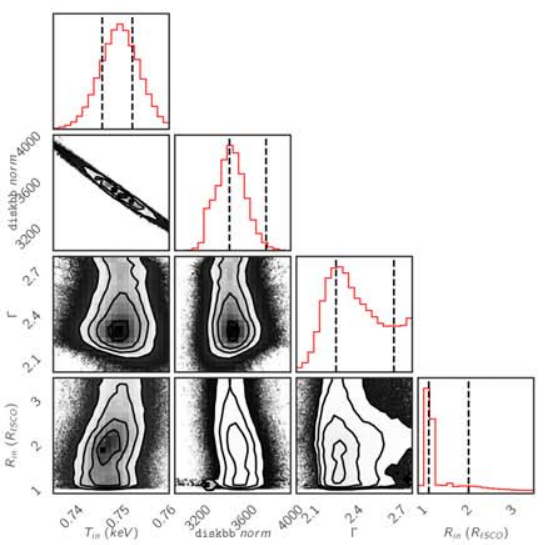

(f) $H R \simeq 0.13$

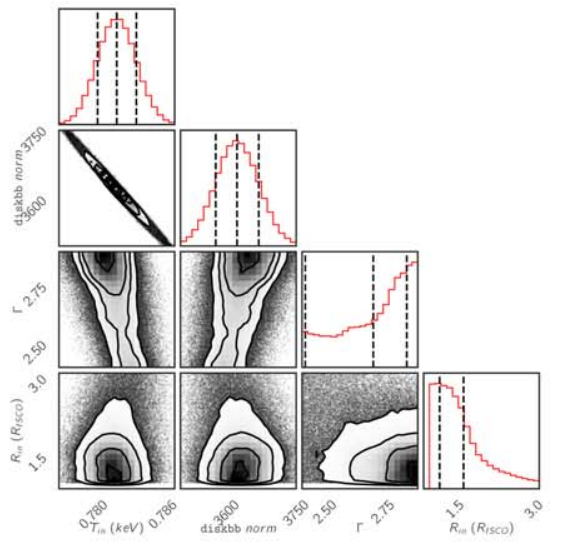

$(\mathrm{g}) H R \simeq 0.05$

Figure 11. One- and two-dimensional projections of the posterior probability distributions, and the $0.16,0.5$, and 0.84 quantile contours derived from the MCMC analysis for the inner disk temperature $\left(T_{\text {in }}\right)$, diskbb norm, photon index $(\Gamma)$, and inner truncation radius $\left(R_{\text {in }}\right)$, measured using reflection spectroscopy for different spectral hardness values across the bright intermediate states of the 2004-2005 outburst. Note that the value of the diskbb norm is frozen to 0 for HR $\simeq 0.8$, $\mathrm{HR} \simeq 0.72$, and $\mathrm{HR} \simeq 0.65$. These figures are produced using the corner package (Foreman-Mackey 2016).

\section{ORCID iDs}

Navin Sridhar (1) https://orcid.org/0000-0002-5519-9550

Javier A. García (i) https://orcid.org/0000-0003-3828-2448

James F. Steiner (1) https://orcid.org/0000-0002-5872-6061

Riley M. T. Connors (i) https://orcid.org/0000-0002-

8908-759X

Victoria Grinberg (ib https://orcid.org/0000-0003-2538-0188

\section{References}

Anders, E., \& Grevesse, N. 1989, Geochim. Cosmochim. Acta, 53, 197

Arnaud, K. A. 1996, in ASP Conf. Serl. 101, Astronomical Data Analysis Software and Systems V, ed. G. H. Jacoby \& J. Barnes (San Francisco, CA: ASP), 17

Balbus, S. A., \& Hawley, J. F. 1991, ApJ, 376, 214

Bardeen, J. M., Press, W. H., \& Teukolsky, S. A. 1972, ApJ, 178, 347

Basak, R., \& Zdziarski, A. A. 2016, MNRAS, 458, 2199 
Begelman, M. C., \& Pringle, J. E. 2007, MNRAS, 375, 1070

Belloni, T., Homan, J., Casella, P., et al. 2005, A\&A, 440, 207

Beloborodov, A. M. 1999, ApJL, 510, L123

Beloborodov, A. M. 2017, ApJ, 850, 141

Bisnovatyi-Kogan, G. S., \& Blinnikov, S. I. 1976, SvAL, 2, 191

Cabanac, C., Fender, R. P., Dunn, R. J. H., et al. 2009, MNRAS, 396, 1415

Chandrasekhar, S. 1960, PNAS, 46, 253

Choudhury, K., García, J. A., Steiner, J. F., et al. 2017, ApJ, 851, 57

Corbel, S., Nowak, M. A., Fender, R. P., et al. 2003, A\&A, 400, 1007

Cowley, A. P., Schmidtke, P. C., Hutchings, J. B., et al. 2002, AJ, 123, 1741

Dauser, T., García, J., Parker, M. L., et al. 2014, MNRAS, 444, L100

Dauser, T., García, J., Walton, D. J., et al. 2016, A\&A, 590, A76

Davis, S. W., Blaes, O. M., Hubeny, I., et al. 2005, ApJ, 621, 372

Davis, S. W., Done, C., \& Blaes, O. M. 2006, ApJ, 647, 525

Davis, S. W., \& El-Abd, S. 2019, ApJ, 874, 23

Del Santo, M., Malzac, J., Jourdain, E., et al. 2008, MNRAS, 390, 227

Done, C., \& Diaz Trigo, M. 2010, MNRAS, 407, 2287

Ebisawa, K. 1991, PhD thesis, Insititute of Space and Astronautical Science/ Japan Aerospace Exploration Agency

Fabian, A. C., Rees, M. J., Stella, L., et al. 1989, MNRAS, 238, 729

Fender, R., \& Belloni, T. 2012, Sci, 337, 540

Fender, R. P., Belloni, T. M., \& Gallo, E. 2004, MNRAS, 355, 1105

Fender, R. P., Homan, J., \& Belloni, T. M. 2009, MNRAS, 396, 1370

Foreman-Mackey, D. 2016, JOSS, 1, 24

Foreman-Mackey, D., Hogg, D. W., Lang, D., et al. 2013, PASP, 125, 306

Fürst, F., Nowak, M. A., Tomsick, J. A., et al. 2015, ApJ, 808, 122

Gallo, E., Corbel, S., Fender, R. P., et al. 2004, MNRAS, 347, L52

García, J., Dauser, T., Lohfink, A., et al. 2014a, ApJ, 782, 76

García, J., Dauser, T., Reynolds, C. S., et al. 2013, ApJ, 768, 146

García, J., \& Kallman, T. R. 2010, ApJ, 718, 695

García, J. A., McClintock, J. E., Steiner, J. F., et al. 2014b, ApJ, 794, 73

García, J. A., Steiner, J. F., McClintock, J. E., et al. 2015, ApJ, 813, 84

García, J. A., Tomsick, J. A., Sridhar, N., et al. 2019, ApJ, 885, 48

Gierliński, M., \& Done, C. 2004, MNRAS, 347, 885

Guilbert, P. W., Fabian, A. C., \& Rees, M. J. 1983, MNRAS, 205, 593

Haardt, F., \& Maraschi, L. 1993, ApJ, 413, 507

Heida, M., Jonker, P. G., Torres, M. A. P., et al. 2017, ApJ, 846, 132

Homan, J., \& Belloni, T. 2005, Ap\&SS, 300, 107

Hynes, R. I., Steeghs, D., Casares, J., et al. 2003, ApJL, 583, L95

Hynes, R. I., Steeghs, D., Casares, J., et al. 2004, ApJ, 609, 317

Igumenshchev, I. V. 2009, ApJL, 702, L72

Jahoda, K., Markwardt, C. B., Radeva, Y., et al. 2006, ApJS, 163, 401

Jahoda, K., Swank, J. H., Giles, A. B., et al. 1996, Proc. SPIE, 2808, 59

Jiang, J., Fabian, A. C., Wang, J., et al. 2019, MNRAS, 484, 1972

Johansen, A., \& Levin, Y. 2008, A\&A, 490, 501

Kara, E., Steiner, J. F., Fabian, A. C., et al. 2019, Natur, 565, 198

Keck, M. L., Brenneman, L. W., Ballantyne, D. R., et al. 2015, ApJ, 806, 149

Kerr, R. P. 1963, PhRvL, 11, 237

King, A. R., \& Ritter, H. 1998, MNRAS, 293, L42

Kubota, A., Tanaka, Y., Makishima, K., et al. 1998, PASJ, 50, 667

Lightman, A. P., \& Zdziarski, A. A. 1987, ApJ, 319, 643

Mahmoud, R. D., Done, C., \& De Marco, B. 2019, MNRAS, 486, 2137

Makishima, K., Maejima, Y., Mitsuda, K., et al. 1986, ApJ, 308, 635

Malzac, J., Beloborodov, A. M., \& Poutanen, J. 2001, MNRAS, 326, 417

Markert, T. H., Canizares, C. R., Clark, G. W., et al. 1973, ApJL, 184, L67

McClintock, J. E., \& Remillard, R. A. 2006, in Black Hole Binaries, ed.

W. Lewin \& M. van der Klis (Cambridge: Cambridge Univ. Press), 157

McClintock, J. E., Shafee, R., Narayan, R., et al. 2006, ApJ, 652, 518

Méndez, M., \& van der Klis, M. 1997, ApJ, 479, 926

Merloni, A., Fabian, A. C., \& Ross, R. R. 2000, MNRAS, 313, 193
Meyer, F., Liu, B. F., \& Meyer-Hofmeister, E. 2000, A\&A, 361, 175

Meyer-Hofmeister, E., Liu, B. F., \& Meyer, F. 2009, A\&A, 508, 329 Miller, J. M., D’A1, A., Bautz, M. W., et al. 2010, ApJ, 724, 1441

Miller, J. M., Fabian, A. C., Reynolds, C. S., et al. 2004, ApJL, 606, L131

Miller, J. M., Homan, J., Steeghs, D., et al. 2006, ApJ, 653, 525

Miller, J. M., Reynolds, C. S., Fabian, A. C., et al. 2008, ApJL, 679, L113

Mirabel, I. F., \& Rodríguez, L. F. 1999, ARA\&A, 37, 409

Mitsuda, K., Inoue, H., Koyama, K., et al. 1984, PASJ, 36, 741

Miyamoto, S., Kimura, K., Kitamoto, S., et al. 1991, ApJ, 383, 784

Miyamoto, S., \& Kitamoto, S. 1991, ApJ, 374, 741

Narayan, R., Igumenshchev, I. V., \& Abramowicz, M. A. 2003, PASJ, 55, L69

Narayan, R., Mahadevan, R., \& Quataert, E. 1998, in Theory of Black Hole Accretion Disks, ed. M. A. Abramowicz, G. Björnsson, \& J. E. Pringle (Cambridge: Cambridge Univ. Press), 148

Narayan, R., \& McClintock, J. E. 2008, NewAR, 51, 733

Parker, M. L., Tomsick, J. A., Kennea, J. A., et al. 2016, ApJL, 821, L6

Parker, M. L., Tomsick, J. A., Miller, J. M., et al. 2015, ApJ, 808, 9

Penna, R. F., McKinney, J. C., Narayan, R., et al. 2010, MNRAS, 408, 752

Petrucci, P. O., Merloni, A., Fabian, A., et al. 2001, MNRAS, 328, 501

Plant, D. S., Fender, R. P., Ponti, G., et al. 2014, MNRAS, 442, 1767

Plant, D. S., Fender, R. P., Ponti, G., et al. 2015, A\&A, 573, A120

Pozdnyakov, L. A., Sobol, I. M., \& Syunyaev, R. A. 1983, ASPRv, 2, 189

Reis, R. C., Fabian, A. C., Ross, R. R., et al. 2008, MNRAS, 387, 1489

Remillard, R. A., \& McClintock, J. E. 2006, ARA\&A, 44, 49

Reynolds, M. T., \& Miller, J. M. 2013, ApJ, 769, 16

Ross, R. R., \& Fabian, A. C. 1993, MNRAS, 261, 74

Ruszkowski, M., \& Fabian, A. C. 2000, MNRAS, 315, 223

Salvesen, G., Miller, J. M., Reis, R. C., et al. 2013, MNRAS, 431, 3510

Schnittman, J. D., Krolik, J. H., \& Noble, S. C. 2013, ApJ, 769, 156

Shakura, N. I., \& Sunyaev, R. A. 1973, A\&A, 24, 337

Shaposhnikov, N., Jahoda, K., Markwardt, C., et al. 2012, ApJ, 757, 159

Shimura, T., \& Takahara, F. 1995, ApJ, 445, 780

Steiner, J. F., García, J. A., Eikmann, W., et al. 2017, ApJ, 836, 119

Steiner, J. F., \& McClintock, J. E. 2012, ApJ, 745, 136

Steiner, J. F., McClintock, J. E., \& Narayan, R. 2013, ApJ, 762, 104

Steiner, J. F., McClintock, J. E., Remillard, R. A., et al. 2010, ApJL, 718, L117

Steiner, J. F., Remillard, R. A., García, J. A., et al. 2016, ApJL, 829, L22

Sunyaev, R. A., \& Truemper, J. 1979, Natur, 279, 506

Swank, J. H. 1999, NuPhS, 69, 12

Tamura, M., Kubota, A., Yamada, S., et al. 2012, ApJ, 753, 65

Tananbaum, H., Gursky, H., Kellogg, E., et al. 1972, ApJL, 177, L5

Tao, L., Tomsick, J. A., Walton, D. J., et al. 2015, ApJ, 811, 51

Thorne, K. S. 1974, ApJ, 191, 507

Tomsick, J. A., Kalemci, E., Kaaret, P., et al. 2008, ApJ, 680, 593

Tomsick, J. A., Yamaoka, K., Corbel, S., et al. 2009, ApJL, 707, L87

van der Klis, M. 1989, ARA\&A, 27, 517

Velikhov, E. 1959, Sov. Phys. JETP, 36, 995

Verner, D. A., Ferland, G. J., Korista, K. T., et al. 1996, ApJ, 465, 487

Wang-Ji, J., García, J. A., Steiner, J. F., et al. 2018, ApJ, 855, 61

Wilkins, D. R., \& Gallo, L. C. 2015, MNRAS, 448, 703

Wilms, J., Allen, A., \& McCray, R. 2000, ApJ, 542, 914

Wu, K., Soria, R., Hunstead, R. W., et al. 2001, MNRAS, 320, 177

Zdziarski, A. A., \& Gierliński, M. 2004, PThPS, 155, 99

Zdziarski, A. A., Gierliński, M., Mikołajewska, J., et al. 2004, MNRAS, 351,791

Zdziarski, A. A., Johnson, W. N., \& Magdziarz, P. 1996, MNRAS, 283, 193 Zdziarski, A. A., Ziółkowski, J., \& Mikołajewska, J. 2019, MNRAS, 488, 1026 Zhang, W., Dovčiak, M., \& Bursa, M. 2019, ApJ, 875, 148

Zhu, Y., Davis, S. W., Narayan, R., et al. 2012, MNRAS, 424, 2504

Życki, P. T., Done, C., \& Smith, D. A. 1999, MNRAS, 309, 561 